\title{
Equivalence of Self-similar and Pseudo-self-similar Tiling Spaces in $\mathbb{R}^{2}$
}

\author{
Betseygail Rand
}

Received: 6 August 2004 / Revised: 11 January 2011 / Accepted: 20 January 2011 /

Published online: 9 February 2011

(C) Springer Science+Business Media, LLC 2011

\begin{abstract}
Given a tiling $T$, one may form a related tiling, called the derived Voronoi tiling of $T$, based on a patch of tiles in $T$. Similarly, for a tiling space $X$, one can identify a patch which appears regularly in all tilings in $X$, and form a derived Voronoi space of tilings, based on that patch.

Each tiling or tiling space is defined with an associated group of rigid motions, $G$. In the case where $G$ is a group of translations, a series of results have been proved demonstrating the equivalence of a tiling to its derived Voronoi tilings. Here we generalize these results: first, to groups which include both translations and rotations, and second, to tiling spaces as well.
\end{abstract}

We say that two tilings, or two tiling spaces, are mutually locally derivable, or MLD, if they are related by local operations. A tiling or tiling space with a high degree of hierarchical structure is called a self-similar tiling or self-similar tiling space, respectively. A tiling or tiling space is pseudo-self-similar if it has a weaker degree of hierarchical structure. Finally, given a tiling, we are interested in the set of all derived Voronoi tilings, and whether it contains two elements related by a similar expansion. If so, the set contains a scaled pair (and the set of derived Voronoi spaces contains a scaled pair of spaces, as well).

In this paper, we show:

1. A tiling and its derived Voronoi tiling are MLD, as are tiling space and its derived Voronoi space.

2. If $T$ or $X$ is self-similar, then its derived Voronoi tiling or derived Voronoi space has a scaled pair.

3. If a derived Voronoi tiling, or derived Voronoi tiling space, contains a scaled pair, then the original tiling or tiling space is pseudo-self-similar.

4. If a tiling or tiling space is pseudo-self-similar, then it is MLD to a self-similar tiling or tiling space.

B. Rand ( $\varangle)$

1000 W Court St, Seguin, TX 78155, USA

e-mail: bg_rand@yahoo.com 


\section{Introduction}

In 2000 and 2001, Natalie Priebe and Boris Solomyak published a series of results on self-similar and pseudo-self-similar tilings [2, 3]. Basically, a tiling $T$ has an associated family of tilings, called the derived Voronoi family, $F(T)$. If this family is highly structured, we say it is $\sigma$-finite. Priebe [2] proved that a self-similar tiling has a $\sigma$ finite family $F(T)$, and that any tiling with a $\sigma$-finite derived Voronoi family must be pseudo-self-similar. Priebe-Solomyak [3] showed that given any pseudo-self-similar tiling of $\mathbb{R}^{2}$, one can construct a self-similar tiling which is locally equivalent to the original.

In these results, tiles are identified if they occur as translates of one another. This precludes the possibility of tilings like the pinwheel tiling, where a given tile appears in infinitely many orientations. At the time of their work, recognizability of aperiodic self-similar tilings (a necessary condition for their results) had only been established for tilings whose tiles are identified under translations. Since then, recognizability has been generalized by Holton et al. [1], in $\mathbb{R}^{2}$, for tilings with rotations. We seek to generalize the results of Priebe and Solomyak to include tilings with rotations. (Furthermore, [1] showed that self-similar tilings are not necessarily recognizable in dimension 3 and higher, and so results here are limited to $\mathbb{R}^{2}$.)

Second, Priebe and Solomyak's results involve tilings; we look to also make statements about tiling spaces. (In particular, a self-similar tiling is a fixed point of a substitution map applied to a substitution tiling space. Although we do not make use of the dynamics of a substitution tiling space here, we phrase results in terms of spaces so that they might be useful in that context.)

Complications arise as we consider tiling spaces with rotations. It turns out that $\sigma$-finiteness is no longer an appropriate notion of structure for a derived Voronoi family. We develop a weaker condition, that the derived Voronoi family contains a scaled pair of tilings to replace being $\sigma$-finite.

In this paper, we show that a tiling $T$, or space $X$, is locally related to all of its derived Voronoi tilings. Second, if $X$ is a self-similar tiling space, then its derived Voronoi family has a scaled pair of orbits. If a given space $X$ has a derived Voronoi family containing a scaled pair of orbits, then $X$ is a pseudo-self-similar space. Finally, given a pseudo-self-similar space, we can construct a self-similar tiling space which is locally equivalent to the original. (All tilings are tilings of $\mathbb{R}^{2}$ with rotations.)

In Sect. 2, we give basic definitions and conditions on tilings, and construct the derived Voronoi family of a tiling and tiling space. In Sect. 3, we state our main results. In Sect. 4, we prove that a tiling space is locally equivalent to its derived Voronoi family. In Sect. 5, we prove that a self-similar space has a scaled pair of orbits. In Sect. 6, we show that if a tiling space has a derived Voronoi family with a scaled pair of orbits, then the tiling space is a pseudo-self-similar space. Finally, in Sect. 7 we show that a pseudo-self-similar space is locally equivalent to a self-similar space. 


\section{Preliminaries}

\subsection{Tilings and Tiling Spaces}

Definition 1 A prototile $\tau$, is a pair $(A, l)$ where $A$ is a compact set in $\mathbb{R}^{2}$ which is the closure of its interior, and $l$ is its label. We say that the support of $\tau$ is $A$, $\operatorname{supp}(\tau)=A$.

Let $\hat{G}$ be the subgroup of the Euclidean group which is generated by rotations and translations (but not reflections.) Let $G$ be any closed subgroup of $\hat{G}$. $G$ acts naturally on prototiles.

Definition 2 Given a set of prototiles $\left\{\tau_{i}\right\}$, a tile $t$ is a pair $\{g \cdot A, l\}$, where $(A, l) \in$ $\left\{\tau_{i}\right\}$ and $g \in G$. $\operatorname{Supp}(t)=g \cdot A$, and the label of $t$ is $l$. A tiling $T$ is a covering of $\mathbb{R}^{2}$ by tiles which overlap only on their boundaries.

Definition 3 Given a tiling $T$, a $T$-patch $\mathbf{P}$ is a finite subset of tiles of $T$. In particular, for a subset $A \subset \mathbb{R}^{2}$ and a tiling $T$, we define the outer patch of $A$ as $[A]^{T}=\{t \in T: \operatorname{Supp}(t) \cap A \neq \emptyset\}$.

We will most often use the outer patch of a point, $[x]^{T}$, where $x \in \mathbb{R}^{2}$, and the outer patch of an open ball of radius $r$, centered at a point $x \in \mathbb{R}^{2}$, i.e. $\left[B_{r}(x)\right]^{T}$. In Sect. 7, we will use $\left[N_{R}(A)\right]^{T}:=\left[\cup_{x \in A} B_{R}(x)\right]^{T}$ to refer to the outer patch of a neighborhood of radius $R$ around a subset $A \subseteq \mathbb{R}^{2}$.

Given a patch $P$, let the radius of the patch be the largest $r$ such that $B_{r}(y) \subset P$ for some $y \in P$.

Let $d_{m}$ be the minimum length of any edge of a tile in $T$. Let $d_{M}:=\left\{\sup \mid x_{1}-\right.$ $x_{2} \mid: x_{1}, x_{2} \in \partial t_{i}$, where $t_{i}$ is any tile in $T$. $\}$ Let $d_{\theta}$ be the minimum angle of any two incoming edges to any vertex.

Two patches $P_{1}$ and $P_{2}$ are equivalent if there is a $g \in G$ such that $P_{1}=g \cdot P_{2}$.

We next define a metric on tilings. Two tilings are close if they match on a large ball around the origin, up to a small action $g \in G$. To be precise, define first the function $\tilde{\rho}$ :

$$
\begin{aligned}
\tilde{\rho}\left(T_{1}, T_{2}\right)= & \inf \left\{\epsilon: \text { there exists } P \subset T_{1} \text { and } Q \subset T_{2} \text { where } B_{1 / \epsilon}(\overrightarrow{0}) \subset\right. \\
& (\operatorname{Supp}(P) \cap \operatorname{Supp}(Q)) \text { and } P=g \cdot Q \text { for some } g \in G \text { with }\|g\|<\epsilon\},
\end{aligned}
$$

where $\|g\|<\epsilon$ if, when written uniquely as its translation and rotational components such that $g \cdot P=|\theta| P+|d|$, both $|\theta|<\epsilon$ and $|d|<\epsilon$. We then define the distance, $\rho$, between two tilings:

$$
\rho\left(T_{1}, T_{2}\right)=\min \left(1, \tilde{\rho}\left(T_{1}, T_{2}\right)\right) .
$$

Definition 4 Given a tiling $T$ and associated group $G$, a tiling space $X$ is the orbit closure of $T$ under $G$ (i.e., the closure under the topology defined by $\rho$, of the orbit of $T$ under $G$ ). 
At times, we will use the notation $\bar{T}$ to emphasize that the tiling space has been formed as the orbit closure of a particular tiling, $T$.

\subsubsection{Useful Conditions on Tiling Spaces}

We restrict our attention to tiling spaces which meet the following conditions:

1. Nonperiodicity: For any $T \in X$, and any infinite order $g \in G$, we have $g \cdot T \neq T$.

2. Almost Periodicity: $X$ is almost periodic if, for any patch $P$ in a tiling $T \in X$, there is a radius $R>0$ such that for any $y \in \mathbb{R}^{2}$ and any $T^{\prime} \in X$, there is a $g \in G$ such that $g \cdot P \subset\left[B_{R}(y)\right]^{T^{\prime}}$.

Definition 5 Given a tiling space $X$, the almost periodicity radius of a patch $P$ is the least $R$ such that for any $y \in \mathbb{R}^{2}$ and any $T \in X$, there is a $g \in G$ such that $g \cdot P \subset\left[B_{R}(y)\right]^{T}$.

In other words, although our tilings never repeat exactly, every patch occurs with statistic regularity. Within any ball of radius $R$, we are guaranteed to find a copy of $P$. Notice that if a tiling space is almost periodic, then every tiling contains every patch that appears in any tiling. This implies that a tiling space is the orbit closure of every tiling it contains.

3. Finite Local Complexity, or FLC: A tiling space $X$ has FLC if for any $R>0$, there is a finite list of patches $P_{1}, \ldots P_{N}$ such that for any $y \in \mathbb{R}^{2}, T \in X,\left[B_{R}(y)\right]^{T}=$ $g \cdot P_{i}$ for some $i \in[1, \ldots, N]$ and $g \in G$.

Lemma 6 If a tiling space has FLC, then it is compact.

Proof Let $\left\{T_{i}\right\}$ be a sequence of tilings in a space $X$. By FLC, there are finitely many possibilities for $\left\{\left[B_{r}(0)\right]^{T}\right\}_{T \in X}$, up to the action of $G$. Therefore, there is a subsequence $\left\{T_{i_{j}}\right\}$ such that $\left[B_{i_{j-1}}(0)\right]^{T_{i j-1}}=g_{i_{j}} \cdot\left[B_{i_{j}}(0)\right]^{T_{i j}}$, and $\left\|g_{i_{j}}\right\|<\epsilon$ for some $\epsilon>0$. Since the group $G$ is compact on regions of bounded translations, we can take a new subsequence of the first subsequence, which converges in $G$ as well. Therefore $\lim _{k \rightarrow \infty} g_{i_{j_{k}}}\left[B_{i_{j_{k}}}(0)\right]^{T}$ is a tiling in $X$.

\subsubsection{Comparing Two Tilings}

In the case of sequences, there is the concept of a sliding block code, enabling the conversion of one sequence into another by local operations. For two tilings, being locally derivable is the analogue of the existence of sliding block codes.

Definition 7 Given two tilings, $T_{1}$ and $T_{2}$, we say that $T_{2}$ is locally derivable from $T_{1}$ if there exists an $R>0$ such that if $\left[B_{R}(x)\right]^{T_{1}}=g \cdot\left[B_{R}(y)\right]^{T_{1}}$ with $x=g \cdot y$, for $g \in G$, then $[x]^{T_{2}}=g \cdot[y]^{T_{2}}$. If both $T_{1}$ and $T_{2}$ are locally derivable from each other, we say they are mutually locally derivable, or MLD.

If $T_{1}$ is locally derivable from $T_{2}$, then the radius of derivability of $T_{1}$ onto $T_{2}$ is the least $R$ for which the definition holds. 
Lemma 8 Being $M L D$ is an equivalence relation.

Proof First, a tiling is trivially MLD with itself. Second, if $T_{1}$ and $T_{2}$ are MLD tilings, then they are each locally derivable from the other, and so the property is symmetric. Finally, if $T_{2}$ is locally derivable from $T_{1}$ with radius $R_{1}$, and $T_{3}$ is locally derivable from $T_{2}$ with radius $R_{2}$, then $T_{3}$ is locally derivable from $T_{1}$ with radius $R_{1}+R_{2}$.

Lemma 9 If $T_{1}$ and $T_{2}$ are MLD tilings, and $T_{1}$ is nonperiodic, almost periodic, and has finite local complexity, then $T_{2}$ has these properties as well.

Proof Suppose that $T_{2}$ is periodic, and let $g \in G$ be an infinite order element such that $g \cdot T_{2}=T_{2}$. Let $T_{1}$ be locally derivable from $T_{2}$ with radius $R$. For all $x \in \mathbb{R}^{2}$, $g \cdot\left[B_{R}(x)\right]^{T_{2}}=\left[B_{R}(g \cdot x)\right]^{T_{2}}$, and so $g \cdot[x]^{T_{1}}=[g \cdot x]^{T_{1}}$. Therefore $T_{1}$ is periodic, which is a contradiction.

Let $T_{2}$ be locally derivable from $T_{1}$ with radius $R^{\prime}$. Let $\left[B_{d}(x)\right]^{T_{2}}$ be a patch in $T_{2}$. Since $T_{1}$ is almost periodic, patches equivalent to $\left[B_{\left(d+R^{\prime}\right)}(x)\right]^{T_{1}}$ appear regularly throughout $T$. If $\left[B_{\left(d+R^{\prime}\right)}\right]^{T_{1}}(y)=g \cdot\left[B_{\left(d+R^{\prime}\right)}(x)\right]^{T_{1}}$ for some element $g \in G$, then $\left[B_{d}(y)\right]^{T_{2}}=g \cdot\left[B_{d}(x)\right]^{T_{2}}$. Thus $\left[B_{d}(x)\right]^{T_{2}}$ occurs throughout $T_{2}$ with the same regularity as $\left[B_{\left(d+R^{\prime}\right)}(x)\right]^{T_{1}}$ occurs in $T$, and so $T_{2}$ is almost periodic.

Finally, again let $T_{1}$ be locally derivable from $T_{2}$ with radius $R$, and $T_{2}$ be locally derivable from $T_{1}$ with radius $R^{\prime}$. Suppose $T_{2}$ lacks finite local complexity: there is a $d>0$ such that $\left\{\left[B_{d}\left(x_{i}\right)\right]^{T_{2}}\right\}$ is an infinite sequence of patches, no two of which are equivalent. Since $T_{1}$ has finite local complexity, $\left\{\left[B_{d+R^{\prime}}\left(x_{i}\right)\right]^{T_{1}}\right\}$ has a finite list of representatives, up to action by $G$. Therefore there are patches $\left[B_{d}\left(x_{i}\right)\right]^{T_{2}}$ and $\left[B_{d}\left(x_{j}\right)\right]^{T_{2}}$ which are not related by an action by $G$ even though $\left[B_{d+R^{\prime}}\left(x_{i}\right)\right]^{T_{1}}=$ $g \cdot\left[B_{d+R^{\prime}}\left(x_{j}\right)\right]^{T_{1}}$, which is a contradiction.

Definition 10 (MLD Tiling Space) Given two tiling spaces $X$ and $Y$ with the same associated group $G$, we say $Y$ is locally derivable from $X$ if there exists a map $f$ : $X \rightarrow Y$ which commutes with the action of the group, and a radius $r$ such that for any $T_{1}, T_{2} \in X,\left[B_{r}(0)\right]^{T_{1}}=\left[B_{r}(0)\right]^{T_{2}}$ implies [0] ${ }^{f\left(T_{1}\right)}=[0]^{f\left(T_{2}\right)}$. Two tiling spaces are MLD if each is locally derivable from the other.

The following theorem will allow us to readily extend many results about tilings to results about the spaces containing them, throughout this paper.

Theorem 11 Let $\bar{T}$ be the space formed by taking the orbit closure of $T$ under a group $G$, and $\bar{S}$ be the space formed by taking the orbit closure of $S$, under the same group $G$. If $T$ and $S$ are $M L D$, then $\bar{T}$ and $\bar{S}$ are $M L D$.

Proof Let $T$ and $S$ be MLD with radius $R$ and define $f(T)=S$. We extend $f$ : $\bar{T} \rightarrow \bar{S}$ as follows: if $T_{1} \in \bar{T}$, then $T_{1}=\lim _{i \rightarrow \infty} g_{i} \cdot T$ for some sequence $\left\{g_{i}\right\} \in G$. Define $f\left(T_{1}\right):=\lim _{i \rightarrow \infty} g_{i} \cdot S$. Note that this limit exists, and is independent of the sequence $\left\{g_{i}\right\}$ : for any $\epsilon>0$, eventually $\rho\left(g_{i} \cdot T, g_{j} \cdot T\right)<\frac{\epsilon}{1+R \epsilon}$, which implies that $\rho\left(g_{i} \cdot S, g_{j} \cdot S\right)<\epsilon$. By construction, $f$ commutes with the action of $G$. 
Suppose $T_{1}=\lim _{i \rightarrow \infty} g_{i} \cdot T$ and $T_{2}=\lim _{j \rightarrow \infty} h_{j} \cdot T$ are tilings in $\bar{T}$, and suppose $\left[B_{R}(0)\right]^{T_{1}}=\left[B_{R}(0)\right]^{T_{2}}$. Then $\forall \epsilon>0$ there exists some $N$ such that $n>N$ implies that $\left[B_{R}(0)\right]^{g_{n} \cdot T}=k_{n} \cdot\left[B_{R}(0)\right]^{h_{n} \cdot T}$, where $\left\|k_{n}\right\|<\epsilon$.

Therefore $\left[B_{R}\left(g_{n} \cdot 0\right)\right]^{T}=g_{n} k_{n} h_{n}^{-1} \cdot\left[B_{R}\left(h_{n} \cdot 0\right)\right]^{T}$, which implies $\left[g_{n} \cdot 0\right]^{S}=$ $g_{n} k_{n} h_{n}^{-1} \cdot\left[h_{n} \cdot 0\right]^{S}$, and so [0] ${ }^{g_{n} \cdot S}=k_{n} \cdot[0]^{h_{n} \cdot S}$ for all $n>N$. We conclude that $[0]^{f\left(T_{1}\right)}=[0]^{f\left(T_{2}\right)}$ as $n \rightarrow \infty$.

Definition 12 Let $\Psi: A \rightarrow \mathbb{R}^{2}$ be a function for some $A \subset \mathbb{R}^{2}$. The map $\Psi$ is locally derivable from a tiling $T$ if there is some radius $r$ such that whenever $\left[B_{r}(x)\right]^{T}=$ $g \cdot\left[B_{r}(y)\right]^{T}$ with $x=g \cdot y, \Psi(x)=g \cdot \Psi(y)$.

\subsubsection{Self-similarity and Pseudo-self-similarity of Tilings and Tiling Spaces}

We next describe tilings with a high degree of hierarchical structure.

Definition 13 A tiling $T$ is self-affine if there exists a linear expansion $\phi: \mathbb{R}^{2} \rightarrow \mathbb{R}^{2}$ such that

1. $\phi(t)$, the image of the support of any tile $t \in T$, is composed of a union of tiles in $T$.

2. If, for two tiles in $T, t_{1}=g \cdot t_{2}$, then $\phi\left(t_{1}\right)=g^{\prime} \cdot \phi\left(t_{2}\right)$, where $g^{\prime}=\phi g \phi^{-1}$, and $\phi\left(t_{i}\right)$ represents the union of tiles in $T$ which compose the image of $t_{i}$ under $\phi$.

We note that if we label the image $\phi\left(t_{i}\right)$ by the label of $t_{i}$, then $\phi(T)$ is also a tiling, composed of supertiles.

In particular, we will be concerned with a subset of self-affine tilings:

Definition 14 A self-affine tiling $T$ is self-similar if all eigenvalues of the expansion map $\phi$ have equal modulus. In this case, we can define the expansion factor $\lambda=$ $\sqrt{|\operatorname{det} \phi|}$.

Definition 15 A self-similar tiling, $T$, with expansion map $\phi$, is recognizable if there is an $R>0$ such that $\left[B_{R}(x)\right]^{T}=g \cdot\left[B_{R}(y)\right]^{T}$ implies $[x]^{\phi(T)}=g \cdot[y]^{\phi(T)}$.

The radius $R$ is called the recognizability radius.

Theorem 16 [1] In dimensions $\leq 2$, self-similar tilings are recognizable.

Next, we define tilings which are almost self-affine and self-similar:

Definition 17 A tiling $T$ is pseudo-self-affine if there exists a linear expansion $\phi$ such that $\phi(T)$ is MLD with $T$. If $\phi$ is a similarity, then $T$ is pseudo-self-similar.

\subsection{Construction of Derived Voronoi Tilings}

First, let "cell" and "tile" be synonymous words, where "cell" is reserved for the tiles of a derived Voronoi tiling. This will simplify discussions involving both a tiling and a derived Voronoi tiling. 
Given a tiling and a patch in that tiling, the derived Voronoi tiling is a new tiling formed which "sees" the frequency of the patch throughout the tiling.

Formally, we first fix a tiling $T$ (with associated group $G$ ), a point $s \in \mathbb{R}^{2}$, and a radius $r>0$. Let $P$ refer to the patch $\left[B_{r}(s)\right]^{T}$. The associated derived Voronoi tiling will be denoted $V_{(T, s, r)}$.

We locate each copy of the patch $P$ throughout $T$, by defining the locator set $L_{(T, s, r)}$ :

$$
\begin{aligned}
L_{(T, s, r)}= & \left\{q \in \mathbb{R}^{2} \text { such that }\left[B_{r}(q)\right]^{T}=g \cdot\left[B_{r}(s)\right]^{T} \text { and } q=g \cdot s,\right. \\
& \text { where } g \in G\} .
\end{aligned}
$$

Note that if $P$ has $m$-fold rotational symmetry, then either the point $s$ will be the locus of symmetry, or there will be $m$ locator dots, all indicating the presence of the same patch. Also note that since $T$ is almost periodic, points in $L_{(T, s, r)}$ occur regularly. The almost periodicity radius, $R_{(T, s, r)}$, of $P$, guarantees that every disc of radius $R_{(T, s, r)}$ will contain an element of $L_{(T, s, r)}$.

The derived Voronoi tiling is formed as the dual of the locator set. Recall that a prototile $t$ is an ordered pair $(A, l)$, where $A$ is a compact set, and $l$ is the associated label. We need to describe the construction of both the compact sets and labels of the prototiles of a derived Voronoi tiling.

Let $p \in L_{(T, s, r)}$. Define $A_{p}$, the compact set containing $p$, by:

$$
A_{p}=\left\{x \in \mathbb{R}^{2} \text { such that } d(x, p) \leq d\left(x, p^{\prime}\right) \text { for all } p^{\prime} \in L_{(T, s, r)}\right\}
$$

This set is compact, and the set of cells will cover $\mathbb{R}^{2}$ and overlap only on their boundaries. Each cell is a convex polytope.

Observe that the edges of the cell are determined by the locations of all neighboring locator dots. If $p, q \in L_{(T, s, r)}$ belong to adjacent cells, then $d(p, q) \leq$ $2\left(R_{(T, s, r)}+d_{M}\right)$. (Recall that the definition of the almost periodicity radius is based on a patch of tiles: for any $y \in \mathbb{R}^{2}$, there is a $g \in G$ such that $g \cdot P \subset\left[B_{R_{(T, s, r)}}(y)\right]$. Therefore, a point on the boundary of two Voronoi cells could be within $\left[B_{R_{(T, s, r)}}(p)\right]$ and $\left[B_{R_{(T, s, r)}}(q)\right]$ but within neither $B_{R_{(T, s, r)}}(p)$ nor $B_{R_{(T, s, r)}}(y)$. Therefore $d(p, q) \leq$ $2\left(R_{(T, s, r)}+d_{M}\right)$.)

Therefore the shape of the Voronoi cell containing the locator dot $p$ is determined by $\left[B_{4\left(R_{(T, s, r)}+d_{M}\right)}(p)\right]^{T}$. By finite local complexity of $T$, there are finitely many shapes of cells in $V_{(T, s, r)}$.

Next we label the cells. One of the key features of a derived Voronoi tiling is that the labels of the prototiles carry significant information about the behavior of the tiling around that prototile. Because of this, the labeling procedure is somewhat involved. 


\section{Labeling the cells of a derived Voronoi tiling}

Let $T$ be a tiling, and let $d$ be a translation in $G$ with $|d|>0$. Since the action is strictly translation, and has no rotation, we will temporarily denote the operation by + .

Definition 18 Let $\left[B_{r}(x)\right]^{T}$ be a patch of a tiling $T$. The translational symmetry of $\left[B_{r}(x)\right]^{T}$ is the smallest absolute value $|d|$, where $d$ is a translation such that $\left[B_{(r-|d|)}(x)\right]^{T}+d=\left[B_{(r-|d|)}(x+d)\right]^{T}$.

Lemma 19 For a tiling $T$ and real number $m$, there is an upper bound on the radius $r$ of patches $\left[B_{r}(x)\right]^{T}$ with translational symmetry of size $|d| \leq m$.

Proof Suppose not. Then there is a sequence of patches $\left[B_{r_{i}}\left(x_{i}\right)\right]^{T}$ with translational symmetry of size $\leq m$, such that $r_{i} \rightarrow \infty$. Since $T$ is compact, we can find a convergent subsequence $\left[B_{r_{i}}\left(x_{i_{j}}\right)\right]^{T}$, and define $T^{\prime}=\lim _{j \rightarrow \infty}\left[B_{r_{i_{j}}}\left(x_{i_{j}}\right)\right]^{T}$. Then $T^{\prime}$ is periodic and in the orbit closure of $T$, which contradicts the requirement that our tiling spaces be aperiodic.

Fix $T$, a point $s$, a radius $r$, and let $R_{(T, s, r)}$ be the almost periodicity radius of $\left[B_{r}(s)\right]^{T}$. Let $M_{(T, s, r)}$ be the least upper bound on the radius of any patch in $T$ with translational symmetry of size $\leq 16\left(R_{(T, s, r)}+d_{M}\right)$. We will use $\tilde{R}_{(T, s, r)}:=$ $M_{(T, s, r)}+5\left(R_{(T, s, r)}+d_{M}\right)$ as the labeling radius for $V_{(T, s, r)}$.

By finite local complexity, there are a finite number of patches $\left\{H_{1}, \ldots, H_{n}\right\}$, where each $H_{i}=\left[B_{\tilde{R}_{(T, s, r)}}\left(p_{i}\right)\right]^{T}$, such that for all $x \in \mathbb{R}^{2},\left[B_{\tilde{R}_{(T, s, r)}}(x)\right]^{T}=g \cdot H_{i}$ for some $g \in G, 1 \leq i \leq n$. We will use these patches $\left\{H_{i}\right\}$ to label our cells. Let a cell containing a locator point $p \in L_{(T, s, r)}$ have label $H_{i}$, where $H_{i}=g \cdot\left[B_{\tilde{R}_{(T, s, r)}}(p)\right]^{T}$ for some $g \in G$.

$V_{(T, s, r)}$ is now a covering of $\mathbb{R}^{2}$ by tiles. (We point out that in Priebe [2], derived Voronoi cells are labeled based on a much smaller radius, defined to be twice the almost periodicity radius of the patch. We are not claiming that our labeling radius is optimal, but it is sufficient to address the complications arising from including rotations. Note that relabeling the cells of a derived Voronoi tilings with a larger labeling radius creates a second tiling which is locally derivable from the first.)

In Sect. 4, we will show that $V_{(T, s, r)}$ and $T$ are MLD. By Lemma 9, it will follow that $V_{(T, s, r)}$ is nonperiodic, almost periodic, and has finite local complexity.

\subsection{Derived Voronoi Families}

In [2], Priebe defined the derived Voronoi family of a tiling to be the set $\left\{V_{(T, \overrightarrow{0}, r)}\right\}_{r>0}$, the set of all derived Voronoi tilings formed by taking patches centered at the origin. We will also use this set, but we need to distinguish it from larger sets of derived Voronoi tilings. We define the derived Voronoi family of a tiling to be

$$
F(T)=\left\{V_{(T, s, r)}: s \in \mathbb{R}^{2}, r>0\right\} .
$$


Fig. 1 Two discs whose outer patches are equivalent

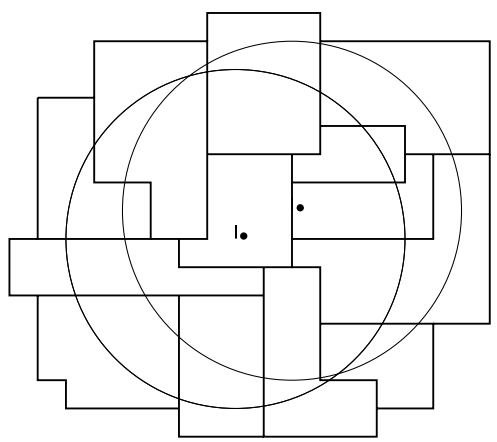

We define the derived Voronoi family of a tiling space, $X$, as

$$
F(X)=\left\{V_{(T, s, r)}: s \in \mathbb{R}^{2}, r>0, T \in X\right\} .
$$

Definition 20 Two tilings, $T$ and $S$, are a scaled pair if there exists a linear expansion $\phi$ such that for every tile $t_{i} \in T, \phi\left(t_{i}\right)$ is a tile in $S$, and $\phi$ induces a bijection between the labels of tiles in $T$ and the labels of tiles in $S$. We denote this $T=\phi S$.

We do not require that $\phi$ be a similarity.

Next, we introduce a hierarchical structure to derived Voronoi families. In [2], the hierarchical structure is that $\left\{V_{(T, \overrightarrow{0}, r)}\right\}_{r>0}$ be $\sigma$-finite. We restate the definition here:

Definition 21 A set of tilings, $F$, is $\sigma$-finite if there exists a finite subset of tilings $\left\{S_{1}, \ldots, S_{n}\right\}$, and a linear expansion $\phi$, such that for every $T \in F, T=\phi^{k}\left(S_{i}\right)$, for some $k$ and $i$.

In [2], it was shown that for self-similar tilings $T$, the associated set $\left\{V_{(T, \overrightarrow{0}, r)}\right\}_{r>0}$ is $\sigma$-finite. However, this property is no longer a reasonable measure of hierarchy when we consider $F(X)$, of a tiling space $X$ that includes rotations. Derived Voronoi tilings built under very similar specifications may look wildly different. We explain this point fully:

Consider two patches in the same tiling, centered $\epsilon$ apart, with the same radius such that $\left[B_{r}(s)\right]^{T}=\left[B_{r}(s+\epsilon)\right]^{T}$. This should be readily available; see Fig. 1 for illustration.

The locator sets $L_{(T, s, r)}$ and $L_{(T, s+\epsilon, r)}$ will both locate the same patches throughout $\mathbb{R}^{2}$. Elements of these locator sets occur in pairs, one from each set, spaced $\epsilon$-apart. (See Fig. 2.)

If $G$ contains an infinite rotation group, then copies of this patch will be identified in infinitely many different orientations. Therefore, the positions of the $\epsilon$-pairs of locator dots will be rotated according to the orientation of the patch containing them. But this will vastly affect the edges of cells in $V_{(T, s, r)}$ and $V_{(T, s+\epsilon, r)}$. (See Fig. 3.) Because of this, the hierarchical structure that a family be $\sigma$-finite is no longer reasonable.

Instead we will use the condition that a derived Voronoi family contains a scaled pair of tilings. 
Fig. 2 Elements of $L_{(T, s, r)}$ and $L_{(T, s+\epsilon, r)}$ occur in pairs
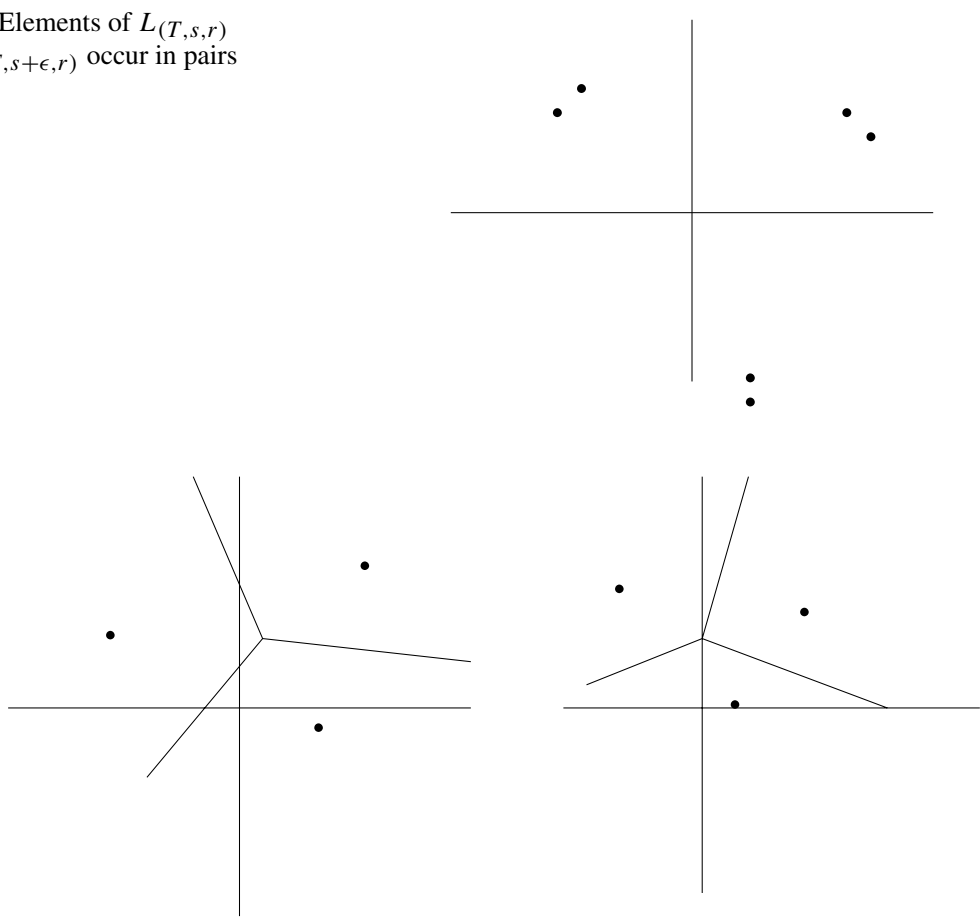

Fig. 3 Edges of cells in $V_{(T, s, r)}$ and $V_{(T, s+\epsilon, r)}$ are vastly affected

\section{Statement of Results}

There are three key findings in [2] and [3] which we seek to generalize. The following were all proved for tilings whose associated group $G$ is a translation group. In [2], Priebe shows that a self-similar tiling has a $\sigma$-finite derived Voronoi family of tilings. Second, any tiling with a $\sigma$-finite derived Voronoi family must be pseudo-self-similar. In [3], Priebe and Solomyak showed that every pseudo-self-similar tiling is MLD to a self-similar tiling.

In this paper, we will prove the following, for tilings and tiling spaces whose associated group $G$ may include both translations and rotations.

Theorem 22 For any patch $\left[B_{r}(s)\right]^{T}, V_{(T, s, r)}$ and $T$ are $M L D$.

Theorem 23 Let $T$ be a self-similar tiling, with associated group $G$. Then the set $\left\{V_{(T, \overrightarrow{0}, r)}\right\}$ is $\sigma$-finite.

Theorem 24 Let $T$ be a self-similar tiling, and $X$ the space formed by taking the orbit closure of $T$ under the associated group $G$. Then both $F(T)$ and $F(X)$ contain a scaled pair of tilings.

Theorem 25 Let $F(T)$ have a scaled pair of tilings. Then $T$ is a pseudo-self-affine tiling. If $\phi$, the expansion map for the scaled pair, is a similarity, then $T$ is a pseudo- 
self-similar tiling. Likewise, let $F(X)$ have a scaled pair. Then $X$ is a pseudo-selfaffine tiling space, and if $\phi$ is a similarity, then $X$ is pseudo-self-similar.

Theorem 26 If $X$ is a pseudo-self-similar tiling space, then it is MLD to a selfsimilar tiling space, $X^{\prime}$.

\section{A Tiling is MLD to Any of Its Derived Voronoi Tilings}

We now prove the close relationship of a tiling to any of its derived Voronoi tilings.

Theorem 22 Let $P=\left[B_{r}(s)\right]^{T}$, and $V_{(T, s, r)}=V_{P}$. Then $V_{P}$ and $T$ are $M L D$.

Proof of Theorem 22 Throughout the proof, let $R_{(T, s, r)}, \tilde{R}_{(T, s, r)}$, and $L_{(T, s, r)}$ be abbreviated by $R_{P}, \tilde{R}_{P}$, and $L_{P}$, respectively, with the understanding that $P=\left[B_{r}(s)\right]^{T}$ is fixed. Recall that $R_{P}$ is the almost periodicity radius of $P, \tilde{R}_{P}$ is the labeling radius of $V_{P}$, and $L_{P}$ is the set of locator dots of $P$ in $V_{P}$.

1. $V_{P}$ is locally derivable from $T$.

Suppose $\left[B_{\left(\tilde{R}_{P}+R_{P}\right)}(x)\right]^{T}=g \cdot\left[B_{\left(\tilde{R}_{P}+R_{P}\right)}(y)\right]^{T}$ and $x=g \cdot y$, for some $g \in G$, and $x, y$ points in $\mathbb{R}^{2}$.

First, assume that there is a unique nearest locator dot to each of $x$ and $y$. Let $p_{x}$ and $p_{y}$ be the nearest locator dots, respectively. Then $\left[B_{\tilde{R}_{P}}\left(p_{x}\right)\right]^{T}=g \cdot\left[B_{\tilde{R}_{P}}\left(p_{y}\right)\right]^{T}$.

Since $\tilde{R}_{P}>2 R_{P}+2 d_{M}$, we know that these patches contain all neighboring locator dots to $p_{x}$ and $p_{y}$, and hence $\left[p_{x}\right]^{V_{P}}$ and $\left[p_{y}\right]^{V_{P}}$ have the same shape. Since the discs around $p_{x}$ and $p_{y}$ match out to the labeling radius $\tilde{R}_{P}$, the cells $\left[p_{x}\right]^{V_{P}}$ and $\left[p_{y}\right]^{V_{P}}$ have the same label. Hence $[x]^{V_{P}}=g \cdot[y]^{V_{P}}$.

If $x$ and $y$ are each equidistant to $n$ locator dots, let $\left\{p_{x_{i}}\right\}$ be the set of locator dots nearest to $x$, and $\left\{p_{y_{i}}\right\}$ the set nearest to $y$, ordered so that $p_{x_{i}}=g \cdot p_{y_{i}}$. Then for each $i,\left[B_{\tilde{R}_{P}}\left(p_{x_{i}}\right)\right]^{T}=g \cdot\left[B_{\tilde{R}_{P}}\left(q_{x_{i}}\right)\right]^{T}$, and so $\left[p_{x_{i}}\right]^{V_{P}}=g \cdot\left[p_{y_{i}}\right]^{V_{P}}$. Therefore $[x]^{V_{P}}=g \cdot[y]^{V_{P}}$.

2. $T$ is locally derivable from $V_{P}$.

We want to show that $T$ is locally derivable from $V_{P}$ with local derivability radius $\tilde{R}_{P}+2 R_{P}$. In other words, that $\left[B_{\left(\tilde{R}_{P}+2 R_{P}\right)}(x)\right]^{V_{P}}=g \cdot\left[B_{\left(\tilde{R}_{P}+2 R_{P}\right)}(y)\right]^{V_{P}}$ and $x=$ $g \cdot y$ imply that $[x]^{T}=g \cdot[y]^{T}$.

First, assume $x$ and $y$ are interior to the cells which contain them. Let $[x]^{V_{P}}$ and [y] $]^{V_{P}}$ each have label $H_{i}$ and contain locator dots $p_{x}$ and $p_{y}$, respectively.

The label $H_{i}$ represents a fixed patch $\left[B_{\tilde{R}_{P}}\left(p_{i}\right)\right]^{T}$, for some $p_{i} \in L_{P}$, and a cell with locator dot $p_{j}$ has label $H_{i}$ if $\left[B_{\tilde{R}_{P}}\left(p_{j}\right)\right]^{T}$ is equivalent to $\left[B_{\tilde{R}_{P}}\left(p_{i}\right)\right]^{T}$. Therefore, $\left[B_{\tilde{R}_{P}}\left(p_{x}\right)\right]^{T}$ and $\left[B_{\tilde{R}_{P}}\left(p_{y}\right)\right]^{T}$ are both equivalent to $\left[B_{\tilde{R}_{P}}\left(p_{i}\right)\right]^{T}$, and so to each other. In other words, we know that $\left[B_{\tilde{R}_{P}}\left(p_{x}\right)\right]^{T}=h \cdot\left[B_{\tilde{R}_{P}}\left(p_{y}\right)\right]^{T}$, for $h \in G$, and we must show that $h=g$.

If the labeling radius were not chosen with sufficient care, it might happen that $h \neq g$. This could happen if a patch of tiles in $T$ gave rise to a patch of cells in 

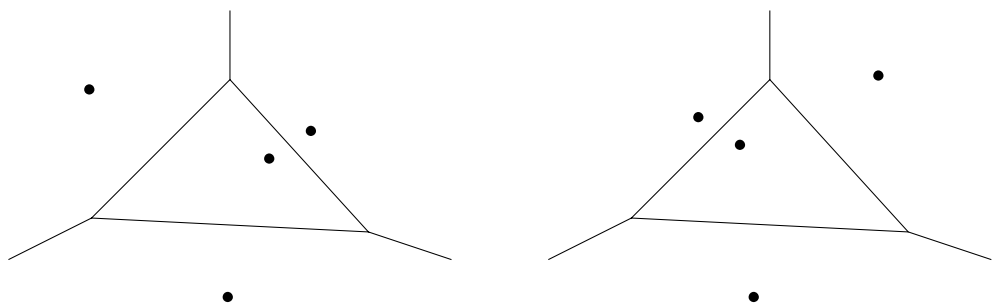

Fig. 4 Two orientations of the same set of locator dots yield indistinguishable cell edges

$V_{P}$, and the patch in $V_{P}$ had rotational symmetry that the patch of tiles in $T$ lacked. For example, in Fig. 4, two orientations of the same set of locator dots give rise to a derived Voronoi cell with rotational symmetry. If $x=g \cdot y$ and $\left[B_{\tilde{R}_{P}}\left(p_{x}\right)\right]^{T}=$ $h \cdot\left[B_{\tilde{R}_{P}}\left(p_{y}\right)\right]^{T}$, but $g \neq h$, then $[x]^{T}$ and $[y]^{T}$ will generally be different tiles.

Let $D_{i}$ be the set of edges which form the dual to $\left.L_{P}\right|_{H_{i}}$, after deleting any infinite edges. We say that an element $h_{x} \in G$ aligns $H_{i}=\left[B_{\tilde{R}_{P}}\left(p_{i}\right)\right]^{T}$ with $[x]^{V_{P}}$ if:

1. $h_{x} \cdot p_{i} \in[x]^{V_{P}}$, and

2. the set of edges of $h_{x} \cdot D_{i}$ is a subset of the set of edges of $\left[B_{\left(\tilde{R}_{P}+2 R_{P}\right)}(x)\right]^{V_{P}}$.

Let $\left\{h_{x}\right\}$ be the set of elements of $G$ which align $H_{i}$ with $[x]^{V_{P}}$, and $\left\{h_{y}\right\}$ be the set of elements in $G$ which align $H_{i}$ with $[y]^{V_{P}}$.

Suppose $\left|\left\{h_{x}\right\}\right|=\left|\left\{h_{y}\right\}\right|=1$. Then $h_{y} h_{x}^{-1}:\left[B_{\tilde{R}_{P}}\left(p_{x}\right)\right]^{V_{P}} \rightarrow\left[B_{\tilde{R}_{P}}\left(p_{y}\right)\right]^{V_{P}}$ uniquely. Therefore $h_{y} h_{x}^{-1}=g$, and so $\left[B_{\tilde{R}_{P}}\left(p_{x}\right)\right]^{T}=g \cdot\left[B_{\tilde{R}_{P}}\left(p_{y}\right)\right]^{T}$. Therefore $[x]^{T}=$ $g \cdot[y]^{T}$.

Suppose $\left|\left\{h_{x}\right\}\right|=\left|\left\{h_{y}\right\}\right|=n>1$. That is, $[x]^{V_{P}}$ and $[y]^{V_{P}}$ have rotational symmetry of order $n$. Let $\left[x^{\prime}\right]^{V_{P}}$ share an edge with $[x]^{V_{P}}$ and have label $H_{j}$. Then $\left[y^{\prime}\right]^{V_{P}}$ shares an edge with $[y]^{V_{P}}$ and also has label $H_{j}$, where $y^{\prime}=g \cdot x^{\prime}$ (since $\left.\left[B_{\left(\tilde{R}_{P}+2 R_{P}\right)}(x)\right]^{V_{P}}=g \cdot\left[B_{\left(\tilde{R}_{P}+2 R_{P}\right)}(y)\right]^{V_{P}}\right)$.

Let $\left\{h_{x^{\prime}}\right\} \in G$ and $\left\{h_{y^{\prime}}\right\} \in G$ be the set of rigid motions that align $H_{j}$ with $\left[x^{\prime}\right]^{V_{P}}$ and $\left[y^{\prime}\right]^{V_{P}}$, respectively. If $\left|\left\{h_{x^{\prime}}\right\}\right|=\left|\left\{h_{y^{\prime}}\right\}\right|=1$, then $h_{y^{\prime}} h_{x^{\prime}}^{-1}:\left[B_{\tilde{R}_{P}}\left(p_{x^{\prime}}\right)\right]^{V_{P}} \rightarrow$ $\left[B_{\tilde{R}_{P}}\left(p_{y^{\prime}}\right)\right]^{V_{P}}$ uniquely. Therefore $h_{y^{\prime}} h_{x^{\prime}}^{-1}=g$, and so $\left[B_{\tilde{R}_{P}}\left(p_{x^{\prime}}\right)\right]^{T}=g \cdot\left[B_{\tilde{R}_{P}}\left(p_{y^{\prime}}\right)\right]^{T}$. Since $x \in\left[B_{\tilde{R}_{P}}\left(p_{x^{\prime}}\right)\right]^{T}$ and $y \in\left[B_{\tilde{R}_{P}}\left(p_{y^{\prime}}\right)\right]^{T}$, we can conclude that $[x]^{T}=g \cdot[y]^{T}$.

Lemma 27 It is not possible that both $\left|\left\{h_{x}\right\}\right|=\left|\left\{h_{y}\right\}\right|>1$ and $\left|\left\{h_{x^{\prime}}\right\}\right|=\left|\left\{h_{y^{\prime}}\right\}\right|>1$.

Assuming the proof of Lemma 27, Theorem 22 follows immediately for any points $x$ and $y$ interior to cells in $V_{P}$. If $x$ and $y$ lie on the boundary of more than one cell, then Lemma 27 implies that one of the cells lacks rotational symmetry. The argument above can be applied using the label of that cell.

During the proof of Lemma 27, we say that $h_{x} \cdot H_{i}$ and $h_{x^{\prime}} \cdot H_{j}$ are consistent if for every $w \in\left(h_{x} \cdot H_{i}\right) \cap\left(h_{x^{\prime}} \cdot H_{j}\right), h_{x}\left[h_{x}^{-1}(w)\right]^{T}=h_{x^{\prime}}\left[h_{x^{\prime}}^{-1}(w)\right]^{T}$. In other words, all edges, vertices and labels of $h_{x} \cdot H_{i}$ match edges, vertices, and labels of $h_{x^{\prime}} \cdot H_{j}$, on their intersection. 
Proof of Lemma 27 A region with two loci of rotational symmetry has a translational symmetry. Suppose $T^{\prime}$ is a tiling with two points of rotational symmetry of the entire plane: rotating $T^{\prime}$ by $\theta_{1}$ around $p_{1}$ maps $T^{\prime}$ to itself, and rotating $T^{\prime}$ by $\theta_{2}$ around $p_{2}$ maps $T^{\prime}$ to itself. Then applying the composition $\theta_{2}^{-1} \theta_{1}^{-1} \theta_{2} \theta_{1}$ to the tiling $T$ results in a translation of length $\leq 4\left|p_{1}-p_{2}\right|$. In other words, $\theta_{2}^{-1} \theta_{1}^{-1} \theta_{2} \theta_{1}(T)=T+d$, where $|d| \leq 4\left|p_{1}-p_{2}\right|$. (For this reason, a tiling cannot have two points of rotational symmetry of the whole plane, because the tiling would then be periodic.)

Suppose $\left|\left\{h_{x}\right\}\right|=\left|\left\{h_{y}\right\}\right|>1$ and $\left|\left\{h_{x^{\prime}}\right\}\right|=\left|\left\{h_{y^{\prime}}\right\}\right|>1$. Let $h_{x_{1}}, h_{x_{2}} \in\left\{h_{x}\right\}$ and $h_{x_{1}^{\prime}}, h_{x_{2}^{\prime}} \in\left\{h_{x^{\prime}}\right\}$. Then there are four consistent pairs: $h_{x_{1}} \cdot H_{i}$ and $h_{x_{1}^{\prime}} \cdot H_{j}, h_{x_{1}} \cdot H_{i}$ and $h_{x_{2}^{\prime}} \cdot H_{j}, h_{x_{2}} \cdot H_{i}$ and $h_{x_{1}^{\prime}} \cdot H_{j}$, and $h_{x_{2}} \cdot H_{i}$ and $h_{x_{2}^{\prime}} \cdot H_{j}$. (There are four more pairs near $y \in \mathbb{R}^{2}$ with which we are not concerned.)

First, $h_{x_{1}^{\prime}} \cdot H_{j}$ is consistent with both $h_{x_{1}} \cdot H_{i}$ and $h_{x_{2}} \cdot H_{i}$. Therefore $\left(h_{x_{1}^{\prime}}\right.$. $\left.H_{j}\right)\left.\right|_{h_{1} \cdot H_{i}}$ has rotational symmetry under $h_{x_{1}} h_{x_{2}}^{-1}$. Let $h_{x_{1}} h_{x_{2}}^{-1}$ be a rotation of angle $\theta_{x}$ around $q_{x}$. Similarly, $h_{x_{1}} \cdot H_{i}$ is consistent with both $h_{x_{1}^{\prime}} \cdot H_{j}$ and $h_{x_{2}^{\prime}} \cdot H_{j}$. Therefore $\left.\left(h_{x_{1}} \cdot H_{i}\right)\right|_{h_{x_{1}^{\prime}} \cdot H_{j}}$ also has rotational symmetry under $h_{x_{1}^{\prime}} h_{x_{2}^{\prime}}^{-1}$. Let $h_{x_{1}^{\prime}} h_{x_{2}^{\prime}}^{-1}$ be a rotation of angle $\theta_{x^{\prime}}$ around $q_{x^{\prime}}$.

Let $F=\left(h_{x_{1}} \cdot H_{i}\right) \cap\left(h_{x_{1}^{\prime}} \cdot H_{j}\right) \cap\left(h_{x_{2}} \cdot H_{i}\right) \cap\left(h_{x_{2}^{\prime}} \cdot H_{j}\right)$. Since the cells $H_{i}$ and $H_{j}$ share an edge in $V_{P}$, we know that $\left|p_{x}-p_{x^{\prime}}\right|<2 R_{P}+2 d_{M}$. Therefore $F$ has radius $>\tilde{R}_{P}-4\left(R_{P}+d_{M}\right)$.

Since $h_{x_{1}} h_{x_{2}}^{-1}:[x]^{V_{P}} \rightarrow[x]^{V_{P}}$, the locus of rotation $q_{x}$ is within $R_{P}+d_{M}$ of the locator point $p_{x}$. Likewise, $\left[x^{\prime}\right]^{V_{P}}$ is fixed under $h_{x_{1}^{\prime}} h_{x_{2}^{\prime}}^{-1}$, and so $q_{x^{\prime}}$ is within $R_{P}+d_{M}$ of $p_{x^{\prime}}$. Therefore the two loci of rotational symmetry of $F$ are at most $4\left(R_{P}+d_{M}\right)$ apart, and so $F$ has translational symmetry of size $\leq 16\left(R_{P}+d_{M}\right)$.

We have, then, that $F$ has radius $>\tilde{R}_{P}-4\left(R_{P}+d_{M}\right)$ in $T$ and translational symmetry of size $\leq 16\left(R_{P}+d_{M}\right)$. Recall that $M_{P}$ is the least upper bound on the radius of any patch in $T$ with translational symmetry of size $\leq 16\left(R_{P}+d_{M}\right)$. Therefore $\tilde{R}_{P}-4\left(R_{P}+d_{M}\right)<M_{P}$. However, by definition $\tilde{R}_{P}=M_{P}+5\left(R_{P}+d_{M}\right)$, which is a contradiction.

\section{A Self-similar Tiling Space has a Scaled Pair of Orbits}

The next two theorems explore the hierarchical structure associated with the derived Voronoi families of a self-similar tiling or a self-similar tiling space.

Recall that there are three variations of families. First, we will use the set $\left\{V_{(T, \overrightarrow{0}, r)}\right.$ : $T$ is fixed, $r>0\}$. (Note that Priebe, in [2], referred to this as the derived Voronoi family of the tiling $T$. We are reserving that term for the next set, and will simply refer to this set as $\left\{V_{(T, \overrightarrow{0}, r)}\right\}$.)

Second, the derived Voronoi family of a tiling $T$ is the set $F(T):=\left\{V_{(T, s, r)}: s \in\right.$ $\left.\mathbb{R}^{2}, r>0\right\}$. Finally, the derived Voronoi family of a tiling space $X$ will refer to the set $F(X):=\left\{V_{(T, s, r)}: T \in X, s \in \mathbb{R}^{2}, r>0\right\}$.

Once we have that $\left\{V_{(T, \overrightarrow{0}, r)}\right\}$ is $\sigma$-finite, it will be immediate to show that $F(T)$ and $F(X)$ both have a scaled pair of tilings.

We now will prove Theorem 23, which we restate here: 
Theorem 23 Let $T$ be a self-similar tiling. Then the set $\left\{V_{(T, \overrightarrow{0}, r)}\right\}$ is $\sigma$-finite.

We wish to emphasize that the first half of this proof-that there are finitely many locator sets up to scale - is a modified version of the proof by Priebe [2], that $\left\{V_{(T, \overrightarrow{0}, r)}\right\}$ is $\sigma$-finite when $G$ is a translation group. We begin with a lemma, which appears first in [2] for tilings defined under a translation group.

Lemma 28 Let $T$ be a self-similar tiling with expansion map $\phi$, and let $\lambda$ be the scaling factor of $\phi$, and let $T$ have recognizability radius $\rho$. Let $l$ be the least integer such that $\lambda^{l}(\lambda-1)>1$. Then the recognizability radius of the image of $T$ under the map $\phi^{k}$ is bounded above by $\lambda^{k+l} \rho$.

Proof of Lemma 28 Because $T$ is recognizable, $\left[B_{\rho}(x)\right]^{T}=g \cdot\left[B_{\rho}(y)\right]^{T}$ with $x=g \cdot y$ implies $[x]^{\phi(T)}=g \cdot[y]^{\phi(T)}$. Applying $\phi^{k-1}$ to the tiling gives us that for all $x, y \in \mathbb{R}^{2},\left[B_{\lambda^{k-1} \rho}(x)\right]^{\phi^{k-1} T}=g \cdot\left[B_{\lambda^{k-1} \rho}(y)\right]^{\phi^{k-1} T}$ with $x=g \cdot y$ implies that $[x]^{\phi^{k} T}=g \cdot[y]^{\phi^{k} T}$. In other words, a radius of $\lambda^{k-1} \rho$ around $x$ is sufficient in $\phi^{k-1} T$ to identify the $k$ th order tile containing $x$. Likewise, a radius of $\lambda^{k-2} \rho$ around $x$ is sufficient in $\phi^{k-2} T$ to identify the $(k-1)$ st order tile containing $x$. Therefore, a radius of $\lambda^{k-1} \rho+\lambda^{k-2} \rho$ is sufficient in $\phi^{k-2} T$ to identify the $k$ th order tile containing $x$.

Continuing to break each tile into smaller pieces, we get that a radius of $\Sigma_{j=0}^{k-1} \lambda^{j} \rho$ around $x$ in $T$ is sufficient to determine which $k$ th order supertile contains $x$. Finally, note that $\Sigma_{j=0}^{k-1} \lambda^{j}=\frac{\lambda^{k}-1}{\lambda-1}<\frac{\lambda^{k}}{\lambda-1}=\frac{\lambda^{k} \lambda^{l}}{(\lambda-1) \lambda^{l}}$. Since $l$ was chosen such that the denominator is greater than 1 , we get $\Sigma_{j=0}^{k-1} \lambda^{j} \rho<\lambda^{k+l} \rho$.

Proof of Theorem 23 Let $E=[\overrightarrow{0}]^{T}$ be the elementary patch containing the origin, and $L_{(T, \overrightarrow{0}, 0)}$ be denoted $L_{E}$. Consider the outer patch of radius $\lambda^{l+1} \rho$ around each locator dot $q \in L_{E}$. By finite local complexity, there is a maximal list $\left\{\left[B_{\lambda^{l+1} \rho}\left(q_{1}\right)\right]^{T}, \ldots,\left[B_{\lambda^{l+1} \rho}\left(q_{n}\right)\right]^{T}\right\}$, such that $\left[B_{\lambda^{l+1} \rho}\left(q_{i}\right)\right]^{T} \neq g \cdot\left[B_{\lambda^{l+1} \rho}\left(q_{j}\right)\right]^{T}$ for any $g \in G, i \neq j$. Let the patch $\left[B_{\lambda^{l+1} \rho}\left(q_{i}\right)\right]^{T}$ be denoted $F_{i}$, and let the locator set $L_{\left(T, q_{i}, \lambda^{l+1} \rho\right)}$ be denoted $L_{F_{i}}$. Subsets of $\left\{L_{F_{1}}, \ldots, L_{F_{n}}\right\}$ will form the locator sets for the finite set of tilings that yield all of $\left\{V_{(T, \overrightarrow{0}, r)}\right\}$, under expansion by powers of $\phi$.

Let $r>0$, and let $k$ be the integer such that $\lambda^{k+l} \rho<r \leq \lambda^{k+l+1} \rho$. (If $r<\lambda^{l+1}$, let $k=0$.) Since $r$ is larger than the recognizability radius of $\phi^{k}$, we have that $q \in$ $L_{(T, \overrightarrow{0}, r)}$ implies that $[q]^{\phi^{k} T}=g \cdot[0]^{\phi^{k} T}=g \cdot \phi^{k}[0]^{T}$ for some $g \in G$. Therefore $q$ is in $\phi^{k} L_{E}$.

Since $L_{E}$ has been partitioned into $\left\{L_{F_{1}}, \ldots, L_{F_{n}}\right\}$, let $\left\{L_{F_{i_{1}}}, \ldots, L_{F_{i_{m}}}\right\}$ be the subset of $L_{E}$ such that $L_{(T, \overrightarrow{0}, r)} \cap \phi^{k} L_{F_{i_{j}}} \neq \emptyset$. We have then that $L_{(T, \overrightarrow{0}, r)} \subseteq \phi^{k}\left\{L_{F_{i_{1}}} \cup\right.$ $\left.\cdots \cup L_{F_{i_{m}}}\right\}$.

Next we show that $\phi^{k}\left\{L_{F_{i_{1}}} \cup \cdots \cup L_{F_{i_{m}}}\right\} \subseteq L_{(T, \overrightarrow{0}, r)}$. Let $q \in \phi^{k} L_{F_{i_{j}}}$ for some $j$. Since $L_{(T, \overrightarrow{0}, r)} \cap \phi^{k} L_{F_{i j}} \neq \emptyset$, let $p$ be a point in the intersection. Thus $\left[B_{r}(p)\right]^{T}=$ $g \cdot\left[B_{r}(\overrightarrow{0})\right]^{T}$ for some $g$, and $\left[B_{\lambda^{k+l+1} \rho}(p)\right]^{T}=g^{\prime} \cdot\left[B_{\lambda^{k+l+1} \rho}(q)\right]^{T}$ for some $g^{\prime}$.

Since $r \leq \lambda^{k+l+1} \rho,\left[B_{r}(q)\right]^{T}=g^{\prime-1} g \cdot\left[B_{r}(\overrightarrow{0})\right]^{T}$, and so $q \in L_{(T, \overrightarrow{0}, r)}$. Therefore $L_{(T, \overrightarrow{0}, r)}=\phi^{k}\left\{L_{F_{i_{1}}} \cup \cdots \cup L_{F_{i_{m}}}\right\}$. Since there are finitely many subsets of 
$\left\{L_{F_{1}}, \ldots, L_{F_{n}}\right\}$, there are finitely many possibilities for locator sets $L_{(T, \overrightarrow{0}, r)}$, up to expansion by a power of $\phi$.

Finally, we must show that this set of locator sets yields finitely many tilings, up to scale by $\phi^{k}$, when we label the derived Voronoi cells. We claim that there is an upper bound on the number of labels of any tiling in $\left\{V_{(T, \overrightarrow{0}, r)}\right\}$.

Lemma 29 For $r>1, \tilde{R}_{(T, \overrightarrow{0}, r)}<C r$ for some constant $C$.

Proof Recall that $\tilde{R}_{(T, \overrightarrow{0}, r)}:=M_{(T, \overrightarrow{0}, r)}+5 R_{(T, \overrightarrow{0}, r)}+5 d_{M}$, where $M_{(T, \overrightarrow{0}, r)}$ is the least radius of a patch with translational symmetry $\leq 16\left(R_{(T, \overrightarrow{0}, r)}+d_{M}\right)$. First we claim that $R_{(T, \overrightarrow{0}, \lambda r)}<\lambda R_{(T, \overrightarrow{0}, r)}$.

Note that $\left[B_{\lambda r}(\overrightarrow{0})\right]^{T} \subset \phi\left[B_{r}(\overrightarrow{0})\right]^{T}$ as subsets of $\mathbb{R}^{2}$, and so $\left[B_{\lambda r}(\overrightarrow{0})\right]^{T} \subset$ $\left[\phi\left[B_{r}(\overrightarrow{0})\right]^{T}\right]^{T}$ as patches in $T$. Therefore the almost periodicity radius of $\left[B_{\lambda r}(\overrightarrow{0})\right]^{T}$ is less than or equal to the almost periodicity radius of $\left[\phi\left[B_{r}(\overrightarrow{0})\right]^{T}\right]^{T}$. While the latter does not lend itself to $R_{(T, \overrightarrow{0}, *)}$ notation, any patch of radius $\lambda R_{(T, \overrightarrow{0}, r)}$ is guaranteed to contain a copy of $\left[\phi\left[B_{r}(0)\right]^{T}\right]^{T}$. We conclude that $R_{(T, \overrightarrow{0}, \lambda r)}<\lambda R_{(T, \overrightarrow{0}, r)}$. Let $k_{1}=\max \left\{\frac{R_{(T, \overrightarrow{0}, d)}}{d}: 0<d \leq \lambda\right\}$. Since $R_{(T, \overrightarrow{0}, r)}$ is an increasing function of $r$, we have $R_{(T, \overrightarrow{0}, r)}<k_{1} r$.

Next we claim that $M_{(T, \overrightarrow{0}, r)}$ grows linearly with $r$. The following proposition is from [1]. A patch is called admissible if it is contained in $T$, and has period $g \in G$ if $P \cap g \cdot P$ is a patch.

Proposition 30 There is a constant $k_{2}>0$ such that if $P$ is an admissible patch whose support contains a ball of radius $r$, then every non-identity period $g$ of $P$ satisfies $\|g b-b\|>k_{2} r$ for some $b \in \operatorname{supp}(P)$.

Clearly, if $g$ is a translation and $P$ is a patch with period $g$, then $\|g b-b\|$ is constant for all $b \in \operatorname{supp}(P)$.

By Proposition 30, if $P$ is an admissible patch whose support contains a ball of radius $M_{(T, \overrightarrow{0}, r)}$, then every translation of $P$ has size $>k_{2} M_{(T, \overrightarrow{0}, r)}$. Since some such patch exists with size $M_{(T, \overrightarrow{0}, r)}$ and translational symmetry $d \leq 16\left(R_{(T, \overrightarrow{0}, r)}+d_{M}\right)$, we have $16\left(R_{(T, \overrightarrow{0}, r)}+d_{M}\right) \geq \vec{d}>k_{2} M_{(T, \overrightarrow{0}, r)}$. Therefore $M_{(T, \overrightarrow{0}, r)}<\left(16 / k_{2}\right)\left(R_{(T, \overrightarrow{0}, r)}+\right.$ $\left.d_{M}\right)<\left(16 / k_{2}\right)\left(k_{1} r+d_{M}\right)$. When $r>1,\left(16 / k_{2}\right)\left(k_{1} r+d_{M}\right)<\left(16 / k_{2}\right)\left(k_{1}+d_{M}\right) r$.

Therefore for $r>1, \tilde{R}_{(T, \overrightarrow{0}, r)}<C r$, where $C=\left(16 / k_{2}\right)\left(k_{1}+d_{M}\right)+5 k_{1}+5 d_{M}$.

We now prove that there is an upper bound on the number of labels of any tiling in $\left\{V_{(T, \overrightarrow{0}, r)}\right\}$. Recall that $\rho$ is the recognizability radius of $T$, and let $N$ be the number of patches of radius $\rho$ in $T$, up to action by $G$. Pick some derived Voronoi tiling $V_{(T, \overrightarrow{0}, s)} \in\left\{V_{(T, \overrightarrow{0}, r)}\right\}$, and let $\left\{q_{1}, \ldots, q_{m}\right\} \in L_{(T, \overrightarrow{0}, s)}$ be a maximal set of locator dots such that every tile in $V_{(T, \overrightarrow{0}, s)}$ is represented by a unique $q_{i}$.

Let $n$ be such that $\rho \lambda^{n-1}<\tilde{R}_{(T, \overrightarrow{0}, s)} \leq \rho \lambda^{n}$, and consider the set of patches $\left\{\left[B_{\lambda^{-n} \tilde{R}_{(T, \overrightarrow{0}, s)}}\left(\phi^{-n} q_{i}\right)\right]^{T}\right\}$. Since $\lambda^{-n} \tilde{R}_{(T, \overrightarrow{0}, s)} \leq \rho$, this set contains at most $N$ distinct elements, up to action by $G$. If $\left[B_{\lambda^{-n} \tilde{R}_{(T, \overrightarrow{0}, s)}}\left(\phi^{-n} q_{i}\right)\right]^{T}=g \cdot\left[B_{\lambda^{-n} \tilde{R}_{(T, \overrightarrow{0}, s)}}\left(\phi^{-n} q_{j}\right)\right]^{T}$ 
for some $i \neq j$, then $\left[B_{\tilde{R}_{(T, \overrightarrow{0}, s)}}\left(q_{i}\right)\right]^{\phi^{n} T}=\phi^{n} g \phi^{-n} \cdot\left[B_{\tilde{R}_{(T, \overrightarrow{0}, s)}}\left(q_{j}\right)\right]^{\phi^{n} T}$. Therefore there are at most $N$ distinct patches in the set $\left\{\left[B_{\tilde{R}_{(T, \overrightarrow{0}, s)}}\left(q_{i}\right)\right]^{\phi^{n^{n}} T}\right\}$ up to action by $G$. It does not follow that $\left[B_{\tilde{R}_{(T, \overrightarrow{0}, s)}}\left(q_{i}\right)\right]^{T}=\phi^{n} g \phi^{-n} \cdot\left[B_{\tilde{R}_{(T, \hat{0}, s)}}\left(q_{j}\right)\right]^{T}$, since there may be several patches in $T$ whose outer patch in $\phi^{n} T$ is composed of the same set of supertiles. We claim that there is a bound on the density of locator dots, and so we can cap the number of possible labels available within $\left[B_{\tilde{R}_{(T, \hat{0}, s)}}\left(q_{i}\right)\right]^{\phi^{n} T}$.

Fix $q_{i}$, and let $\left\{h_{1}, \ldots, h_{z}\right\} \in G$ be all distinct elements such that $h_{i} \cdot\left[B_{s}(\overrightarrow{0})\right]^{T}$ is admissible and contained within $\left[B_{\tilde{R}_{(T, 0, s)}}\left(q_{i}\right)\right]^{\phi^{n} T}$.

Claim: $256 \pi M^{2} / k_{2}^{3}$ is a (crude) upper bound on $z$, where $M=\left(C+C \lambda^{2} d_{M} / \rho\right)$, and $C$ and $k_{2}$ are from above. (The following is due to James B. Shearer, personal communication, August 2010.)

First, $\left[B_{\tilde{R}_{(T, \overrightarrow{0}, s)}}\left(q_{i}\right)\right]^{\phi^{n} T}$ is contained within the disk $B_{\tilde{R}_{(T, \overrightarrow{0}, s)}+\lambda^{n+1} d_{M}}\left(q_{i}\right)$, and $\tilde{R}_{(T, \overrightarrow{0}, s)}+\lambda^{n+1} d_{M}<C s+\lambda^{2} C s d_{M} / \rho=s\left(C+C \lambda^{2} d_{M} / \rho\right)=s M$. Next, $B_{s M}\left(q_{i}\right)$ is contained in the square of side $2 s M$, centered at $q_{i}$.

Suppose $z>256 \pi M^{2} / k_{2}^{3}$. If we can show that there are two patches, $h_{i} \cdot\left[B_{S}(\overrightarrow{0})\right]^{T}$ and $h_{j} \cdot\left[B_{s}(\overrightarrow{0})\right]^{T}$, such that $\left\|h_{i} \cdot \overrightarrow{0}-h_{j} \cdot \overrightarrow{0}\right\|<k_{2} s / 2$, with relative angle $\theta<k_{2} / 2$, then we will have demonstrated a contradiction. (If $g: h_{i} \cdot\left[B_{s}(\overrightarrow{0})\right]^{T} \rightarrow h_{j} \cdot\left[B_{s}(\overrightarrow{0})\right]^{T}$, then for all $b \in h_{i} \cdot\left[B_{s}(\overrightarrow{0})\right]^{T},\|g b-b\|<k_{2} s / 2+s\left(k_{2} / 2\right)$, which violates Proposition 30.)

If there are more than $4 \pi / k_{2}$ images of $\left\{h_{i} \cdot\left[B_{S}(\overrightarrow{0})\right]^{T}\right\}$ contained within a square of side length $k_{2} s / 4$, then all of them will have centers which are at most $k_{2} s / 2$ apart, and at least two of them will have relative angle $<\frac{2 \pi}{4 \pi / k_{2}}=k_{2} / 2$.

There are at most $64 M^{2} / k_{2}^{2}$ non-overlapping squares of side length $k_{2} s / 4$ contained within our square of side length $2 s M$, centered at $q_{i}$. Therefore, we cannot have $\left(64 M^{2} / k_{2}^{s}\right)\left(4 \pi / k_{2}\right)$ images of $\left\{h_{i} \cdot\left[B_{s}(\overrightarrow{0})\right]^{T}\right\}$ within our square of side length $2 s M$, and so we conclude that $z \leq 256 \pi M^{2} / k_{2}^{3}$.

In total, $V_{(T, \overrightarrow{0}, s)}$ must have fewer than $256 \pi M^{2} N / k_{2}^{3}$ labels, and so this bound does not depend on choice of $s$.

Consider all possible subsets of $\left\{L_{F_{1}}, \ldots, L_{F_{n}}\right\}$ that are actually realized by some $L_{(T, \overrightarrow{0}, r)}=\phi^{k}\left(L_{F_{i_{1}}} \cup \cdots \cup L_{F_{i_{m}}}\right)$. For each subset, there is a finite number of ways to label the cells with fewer than $256 \pi M^{2} N / k_{2}^{3}$ labels. Therefore we have finitely many derived Voronoi tilings, up to expansion by $\phi^{k}$. This concludes the proof of Theorem 23.

As described in Sect. 2.3, $F(T)$ and $F(X)$ are not necessarily $\sigma$-finite if the associated group contains rotations. However, since $\left\{V_{(T, \overrightarrow{0}, r)}\right\} \subset F(T) \subset F(X)$, both $F(T)$ and $F(X)$ have a scaled pair of derived Voronoi tilings. Therefore this theorem follows immediately:

Theorem 24 Let $T$ be a self-similar tiling, and $X$ the space formed by taking the orbit closure of $T$ under a group $G$. Then both $F(T)$ and $F(X)$ contain a scaled pair of derived Voronoi tilings. 


\section{A Scaled Pair of Voronoi Tilings or Orbits Implies Pseudo-self-similarity}

In this section, we do not require that a tiling $T$ be self-similar, or that a space $X$ be a self-similar tiling space. Instead, we start with the condition that $F(T)$ and $F(X)$ have a scaled pair of derived Voronoi tilings. We will show that if $F(T)$ and $F(X)$ each have a scaled pair, then $T$ and $X$, respectively, are pseudo-self-affine or pseudoself-similar.

We restate Theorem 25 for convenience:

Theorem 25 Let $F(T)$ have a scaled pair. Then $T$ is a pseudo-self-affine tiling. If $\phi$, the expansion map for the scaled pair, is a similarity, then $T$ is a pseudo-self-similar tiling. Likewise, let $F(X)$ have a scaled pair. Then $X$ is a pseudo-self-affine tiling space, and if $\phi$ is a similarity, then $X$ is pseudo-self-similar.

Proof $F(T)$ contains tilings $V_{\left(T, s_{1}, r_{1}\right)}$ and $V_{\left(T, s_{2}, r_{2}\right)}$ where $V_{\left(T, s_{1}, r_{1}\right)}=\phi\left(V_{\left(T, s_{2}, r_{2}\right)}\right)$, and $\phi$ is a linear expansion. From Theorem 22, we have that $T$ is MLD to both of these derived Voronoi tilings.

Since $T$ is MLD to $V_{\left(T, s_{2}, r_{2}\right)}, \phi(T)$ is $\operatorname{MLD}$ to $\phi\left(V_{\left(T, s_{2}, r_{2}\right)}\right)$. Because $\phi\left(V_{\left(T, s_{2}, r_{2}\right)}\right)=V_{\left(T, s_{1}, r_{1}\right)}$, and $V_{\left(T, s_{1}, r_{1}\right)}$ is MLD to $T$, we have that $\phi(T)$ is MLD to $T$. Therefore $T$ is pseudo-self-affine, and if $\phi$ is a similarity, then $T$ is pseudo-selfsimilar.

Schematically:

$$
\begin{aligned}
V_{\left(T, s_{1}, r_{1}\right)} \stackrel{\mathrm{MLD}}{\longleftrightarrow} \begin{array}{c}
T \\
\text { Applying } \phi:
\end{array} & \stackrel{\mathrm{MLD}}{\longleftrightarrow} V_{\left(T, s_{2}, r_{2}\right)} \\
\phi(T) & \stackrel{\mathrm{MLD}}{\longleftrightarrow} \phi\left(V_{\left(T, s_{2}, r_{2}\right)}\right)=V_{\left(T, s_{1}, r_{1}\right)} \stackrel{\mathrm{MLD}}{\longleftrightarrow} T .
\end{aligned}
$$

Finally, suppose $F(X)$ contains a scaled pair $V_{\left(T_{1}, s_{1}, r_{1}\right)}=\phi \cdot V_{\left(T_{2}, s_{2}, r_{2}\right)}$. Then we can derive an analogous diagram for the spaces formed as the orbit closures of each space:

$$
\begin{aligned}
\bar{V}_{\left(T_{1}, s_{1}, r_{1}\right)} \stackrel{\mathrm{MLD}}{\longleftrightarrow} \bar{T}_{1}=X=\bar{T}_{2} \stackrel{\mathrm{MLD}}{\longleftrightarrow} \bar{V}_{\left(T_{2}, s_{2}, r_{2}\right)} \\
\text { Applying } \phi: \\
\quad \phi(X) \quad \stackrel{\mathrm{MLD}}{\longleftrightarrow} \phi\left(\bar{V}_{\left(T_{2}, s_{2}, r_{2}\right)}\right)=\bar{V}_{\left(T_{1}, s_{1}, r_{1}\right)} \stackrel{\mathrm{MLD}}{\longleftrightarrow} X .
\end{aligned}
$$

\section{Pseudo-self-similarity Implies MLD to a Self-similar Thing}

Theorem 26 If $T$ is a pseudo-self-similar tiling, then it is MLD to a self-similar tiling, $T^{\prime}$. If $X$ is a pseudo-self-similar tiling space, then it is MLD to a self-similar tiling space.

We wish to emphasize that this proof must be essentially credited to PriebeSolomyak in [3]. Here we modify the details to account for a group $G$ involving rotations, and a tiling space $X$.

Let $\partial T$ refer to the boundary graph composed of edges and vertices of tiles in $T$, and let the degree of a vertex refer to the number of edges extending from it. 


\section{Outline of Proof of Theorem 26}

1. Without loss of generality, we can assume our tiling space has certain simplifying properties.

2. If $T$ is pseudo-self-similar with expansion $\phi$, then there is a one-to-one, onto map $\Psi: \partial T \rightarrow \partial\left(\phi^{-k} T\right)$, which maps edges in a piecewise linear, local manner.

3. We may construct a new map $\Psi_{n}:=\phi^{-n k}\left(\phi^{k} \Psi\right)^{n}$, which approximates edges in $T$ with unions of edges in $\phi^{-n k} T$. Taking the $\lim _{n \rightarrow \infty} \Psi_{n}:=\Psi_{\infty}$, we show that $\Psi_{\infty}$ is a continuous, injective map from $\partial T$ to $\mathbb{R}^{2}$.

4. We construct a tiling whose edges and vertices are the image of $T$ under $\Psi_{\infty}$. We call this tiling $T_{\infty}$ and show that $T_{\infty}$ is MLD to $T$.

5. $T_{\infty}$ is a self-similar tiling with expansion $\phi^{k}$, and so $\bar{T}_{\infty}$ is a self-similar tiling space which is MLD to $X$.

\section{Step 1. Simplifying assumptions for a tiling space $X$}

Lemma 31 Let $X$ be a pseudo-self-similar tiling space. Then $X$ is MLD to a pseudoself-similar space $\tilde{X}$, where all tiles are convex polytopes, and each vertex of $\tilde{X}$ has degree 3.

Proof Let $X$ have associated group $G$, and let $T \in X$. Then $V_{(T, s, r)}$ and $T$ are MLD, and $V_{(T, s, r)}$ has the property that all cells are convex polytopes. Let $\bar{V}_{(T, s, r)}$ be the orbit closure of $V_{(T, s, r)}$ under $G$. Then $\bar{V}_{(T, s, r)}$ is a tiling space which satisfies the first property.

We will form a second tiling space, $\bar{V}_{(T, s, r)}^{\prime}$, which is MLD to $\bar{V}_{(T, s, r)}$, and satisfies the second property, as follows: Let $\left\{x_{i}\right\}$ be the set of vertices in $V_{(T, s, r)}$ with degree $>3$. To define $V_{(T, s, r)}^{\prime}$, first let $\partial V_{(T, s, r)}^{\prime}:=\partial V_{(T, s, r)}$ on $\mathbb{R}^{2} \backslash \cup\left\{\left[B_{e / 3}\left(x_{i}\right)\right]^{V_{(T, s, r)}}\right\}$, where $e$ is the minimum length of any edge in $V_{(T, s, r)}$. Each cell of $V_{(T, s, r)}$ whose vertices all have degree $\leq 3$ has been left unchanged in $V_{(T, s, r)}^{\prime}$, and we preserve the label of these cells, as well. In other words, away from every vertex of degree greater than 3 , our new tiling agrees with the old tiling.

Let $\left\{x_{1}, \ldots, x_{n}\right\} \subset\left\{x_{i}\right\}$ be a finite set of unique representatives, such that for all $\left\{x_{i}\right\},\left[B_{e / 3}\left(x_{i}\right)\right]^{V_{(T, s, r)}}=g \cdot\left[B_{e / 3}\left(x_{k}\right)\right]^{V_{(T, s, r)}}$, for $1 \leq k \leq n$, and $g \in G$. We will first define $V_{(T, s, r)}^{\prime}$ for the regions of $\mathbb{R}^{2}$ covered by $\left\{\left[B_{e / 3}\left(x_{k}\right)\right]^{V_{(T, s, r)}}\right\}, 1 \leq k \leq n$, and then extend it by the $g$-action to all regions $\left\{\left[B_{e / 3}\left(x_{i}\right)\right]^{V_{(T, s, r)}}\right\}, i>n$.

Consider $x_{k}, 1 \leq k \leq n$. For each edge extending from $x_{k}$, introduce a vertex at a distance of $e / 3$ from $x_{k}$. Connect each of these new vertices to new vertices on either side, creating a polygon containing $x_{k}$. (See Fig. 5.) Lastly, we delete $x_{k}$ and each edge extending from $x_{k}$ to the new vertices. In doing so, we have created multiple new cells, for each of which we create a new label. If $\left[B_{e / 3}\left(x_{i}\right)\right]^{V_{(T, s, r)}}$ had rotational symmetry, then the new edges of $\left[B_{e / 3}\left(x_{i}\right)\right]^{V_{(T, s, r)}^{\prime}}$ do as well, and we label the new cells to preserve that symmetry.

Finally, we extend this procedure to all $\left[B_{e / 3}\left(x_{i}\right)\right]^{V_{(T, s, r)}}, x_{i}$ a vertex of degree $>3$. If $\left[B_{e / 3}\left(x_{i}\right)\right]^{V_{(T, s, r)}}=g \cdot\left[B_{e / 3}\left(x_{k}\right)\right]^{V_{(T, s, r)}}, 1 \leq k \leq n$, define $\left[B_{e / 3}\left(x_{i}\right)\right]^{V_{(T, s, r)}^{\prime}}=$ $g \cdot\left[B_{e / 3}\left(x_{k}\right)\right]^{V_{(T, s, r)}^{\prime}}$. We label these cells in accordance with the label of the cell 

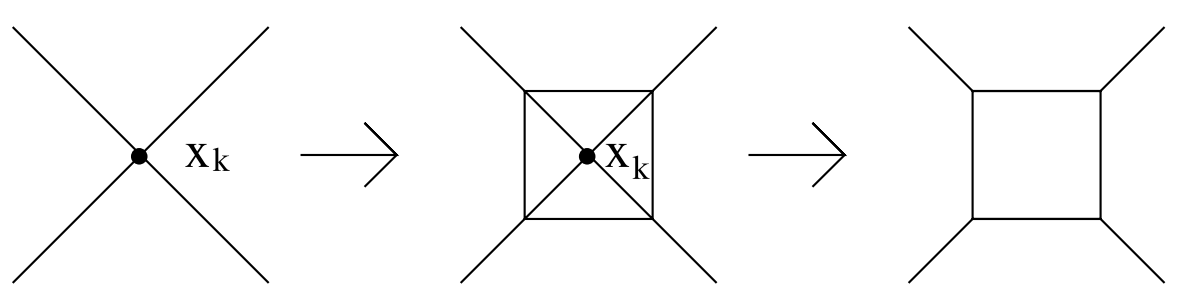

Fig. 5 Local replacement procedure to create MLD tiling where all vertices are of degree 3

related by $g$, noting that if $g$ is not unique, then the labels are the same across all possible choices of $g$.

Note that since this procedure is reversible, $V_{(T, s, r)}$ and $V_{(T, s, r)}^{\prime}$ are MLD, and that $V_{(T, s, r)}^{\prime}$ satisfies property 2. Finally, since $T$ is pseudo-self-similar, $T$ and $\phi(T)$ are MLD. Since $T$ is MLD with $V_{(T, s, r)}^{\prime}$, and $\phi T$ is MLD with $\phi V_{(T, s, r)}^{\prime}$, we have that $V_{(T, s, r)}^{\prime}$ and $\phi V_{(T, s, r)}^{\prime}$ are MLD. Therefore $V_{(T, s, r)}^{\prime}$ is pseudo-self-similar.

Therefore $\bar{V}_{(T, s, r)}^{\prime}$ is MLD to $X$, and satisfies the properties of Lemma 31 .

Our next simplifying assumption states that, for any radius $r$, we can assume that all loci of rotational symmetry of size $r$ are interior to the tiles containing them.

Lemma 32 Let $X$ be a pseudo-self-similar tiling space. Then for any $r>0, X$ is $M L D$ to a pseudo-self-similar space $\hat{X}$, with the following property: for all $\hat{T} \in \hat{X}$, if $\left[B_{r}(x)\right]^{\hat{T}}=g \cdot\left[B_{r}(x)\right]^{\hat{T}}$ with $x=g \cdot x$, then $x \notin \partial \hat{T}$.

Proof We create $\hat{X}$ with a local replacement procedure much like as in the proof of Lemma 31. Let $X$ satisfy the conditions of Lemma 31, and let $T \in X$. Let $\left\{x_{i}\right\}$ be the set of points contained in $\partial T$ such that $\left[B_{r}\left(x_{i}\right)\right]^{T}=g \cdot\left[B_{r}\left(x_{i}\right)\right]^{T}$ with $x_{i}=g \cdot x_{i}$, for some nontrivial $g \in G$.

On $\mathbb{R}^{2} \backslash \cup\left[x_{i}\right]^{T}$, define $\partial \hat{T}:=\partial T$. Any tile not containing an edge or vertex which is a locus of rotational symmetry is left unchanged, and we label these tiles in $\hat{T}$ with the same label as they had in $T$.

Let $\left\{x_{1}, \ldots x_{n}\right\} \subset\left\{x_{i}\right\}$ be a finite set of representatives such that $\left[B_{r}\left(x_{i}\right)\right]^{T} \neq g$. $\left[B_{r}\left(x_{j}\right)\right]^{T}$ with $x_{i}=g \cdot x_{j}$, for $i \neq j, g \in G$.

If $x_{i}$ is a vertex in $T$, then $\left[B_{r}\left(x_{i}\right)\right]^{T}$ must have rotational symmetry of angle $2 \pi / 3$. We replace it with a triangular cell, as depicted in Fig. 5, and label the newly created cells so as to preserve the rotational symmetry. If $x_{i}$ lies on an edge in $T$, then $\left[B_{r}\left(x_{i}\right)\right]^{T}$ must have rotational symmetry of angle $\pi$. We introduce two new chords, related by a rotation of $\pi$ around $x_{i}$, across each tile of which $x_{i}$ is on the boundary. Now $x_{i}$ is a vertex of degree 4 , and we have created two new vertices of degree 3 opposite $x_{i}$. (If the new chords happen to land on existing vertices, offset the new chords by some angle $\theta$, symmetrically on both sides of $x_{i}$.) (See Fig. 6.) We apply the local replacement procedure depicted in Fig. 5 to the vertex $x_{i}$. Note that the result is still symmetric with respect to rotation by $\pi$. We create a new label for each new cell that has been created, keeping the result symmetric with respect to rotation by $\pi$. 

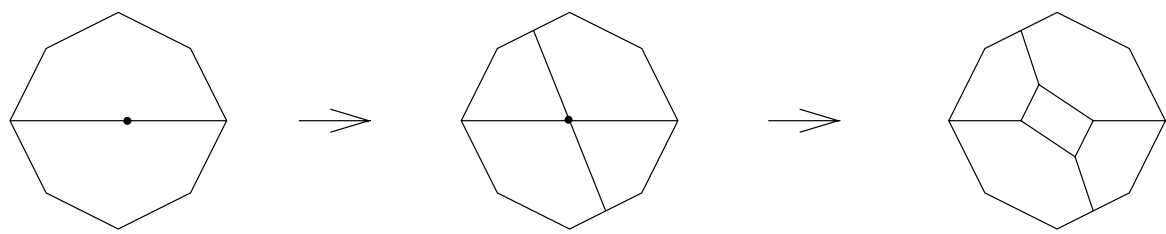

Fig. 6 Local replacement to create tiling where loci of rotational symmetry do not occur within an edge

We now define $\hat{T}$ on $\left\{\left[x_{i}\right]^{T}\right\}$. For each $x_{i},\left[B_{r}\left(x_{i}\right)\right]^{T}=g \cdot\left[B_{r}\left(x_{k}\right)\right]^{T}$, for some $1 \leq k \leq n$ and $g \in G$. Define $\left[B_{e / 3}\left(x_{i}\right)\right]^{\hat{T}}:=g \cdot\left[B_{e / 3}\left(x_{k}\right)\right]^{\hat{T}}$, and label accordingly. (Note that $g$ is not unique, since these are loci of symmetry, but that the replacement procedure respects the symmetry. Also, $\left[x_{i}\right]^{T}$ and $\left[x_{j}\right]^{T}$ may not be disjoint, in which case $\hat{T}$ might lack some rotational symmetry of size $r$ that $T$ has.) As before, since the procedure is reversible, the tiling $\hat{T}$ is MLD to $T$, and so the closure of $\hat{T}$ under $G$ is a tiling space that satisfies the lemma.

\section{Step 2. Developing $\Psi: \partial T \rightarrow \partial\left(\phi^{-k} T\right)$}

Given two points, $x$ and $y$, let $\overrightarrow{x, y}$ be the line segment between $x$ and $y$. For any $k>0$, let $R_{k}$ be the radius of derivability of $\phi^{-k} T$ from $T$.

Recall that $d_{m}$ is the minimum length of any edge of a tile in $T$, that $d_{M}:=$ $\left\{\sup \left|x_{1}-x_{2}\right|: x_{1}, x_{2} \in \partial t_{i}\right.$, where $t_{i}$ is any tile in $\left.T\right\}$, and that $d_{\theta}$ is the minimum angle of any two incoming edges to any vertex in $T$.

Let $K$ be a fixed integer such that $\lambda^{-K}<\frac{d_{m}}{48 d_{M}} \sqrt{2-2 \cos d_{\theta}}$.

Lemma 33 Given a tiling $T$, it is sufficient to prove Theorem 26 holds for tilings MLD to $T$, satisfying the conditions in Lemmas 31 and 32 , with $r=R_{K}$, the radius of derivability of $\phi^{-K} T$ from $T$.

Proposition 34 Let $T$ be a pseudo-self-similar tiling with expansion $\phi$, and $\lambda=|\phi|$, satisfying the conditions in Lemma 33. Let $\epsilon>0$. For all integers $k$ sufficiently large, there is a continuous, one-to-one map $\Psi: \partial T \rightarrow \partial\left(\phi^{-k} T\right)=\phi^{-k} \partial T$ which takes edges in $\partial T$ onto unions of edges in $\phi^{-k} \partial T$ and which satisfies:

1. For $x \in \partial T,|x-\Psi(x)|<\epsilon$.

2. If $l$ is an edge in $\operatorname{Im} \Psi$, then the restriction $\left.\Psi\right|_{\Psi^{-1}(l)}$ is linear. Furthermore, there is a $\rho \in(0,1)$ such that if $e$ is an edge in $T$, and $l \in \Psi(e)$, then $\left|\Psi^{-1}(l)\right| \leq \rho|e|$.

3. The map $\Psi$ is locally derivable from $T$ with radius $\tilde{R}$, for some $\tilde{R}>0$.

Proof of Proposition 34 Without loss of generality, we can assume that:

1. $\epsilon<\frac{d_{m}}{4}$

2. $K<k$

3. $\lambda^{-k}<\frac{\epsilon}{12 d_{M}} \sqrt{2-2 \cos d_{\theta}}$.

Note that $K<k$ implies that all symmetry of size $R_{k}$ has been disrupted. Also, since $0 \leq d_{\theta} \leq 2 \pi / 3$, it follows that $\lambda^{-k} d_{M}<\frac{\epsilon}{6}$. 


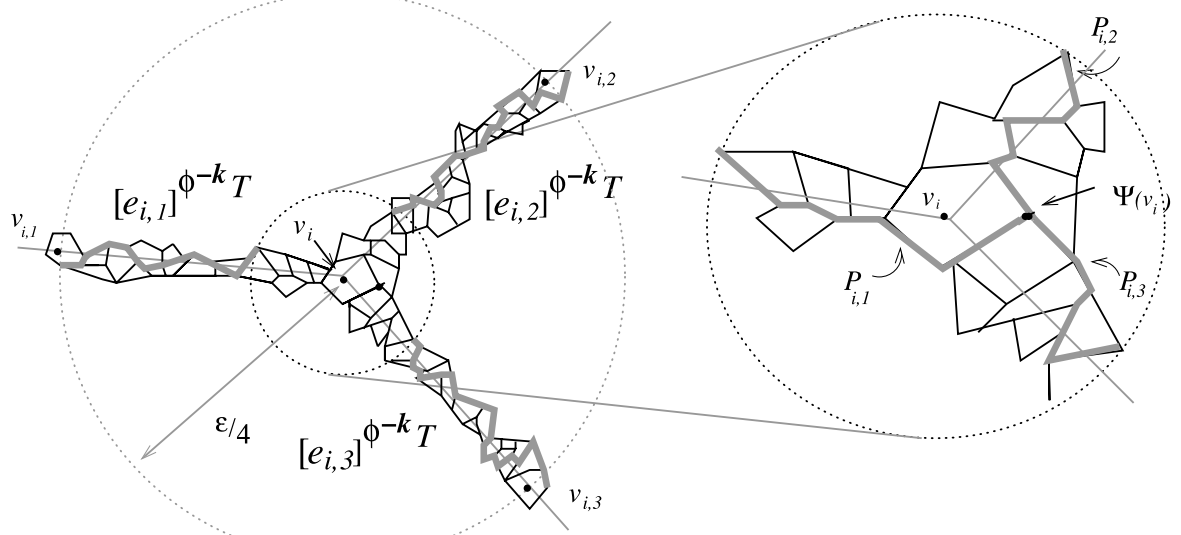

Fig. 7 Notation for incoming edges to $v_{i}$ and $\Psi\left(v_{i}\right)$

\section{Defining $\Psi$ near vertices in $T$}

We partition the vertices of $T$ according to $R_{k}+\epsilon / 4$ : let $\left\{v_{1}, v_{2}, \ldots, v_{m}\right\}$ be a maximal list of vertices such that $\left[B_{R_{k}+\epsilon / 4}\left(v_{i}\right)\right]^{T} \neq g \cdot\left[B_{R_{k}+\epsilon / 4}\left(v_{j}\right)\right]^{T}$ for any $i \neq$ $j, g \in G$. We will define $\Psi$ on each $B_{\epsilon / 4}\left(v_{i}\right) \cap \partial T$, and then extend $\Psi$ to all $\epsilon / 4$ neighborhoods of vertices in $T$ by the action of $G$.

Let $e_{i, 1}, e_{i, 2}$, and $e_{i, 3}$ be the three incoming edges to $v_{i}$ in $T$ and let the point in the intersection of $e_{i, j}$ and $\partial B_{\epsilon / 4}\left(v_{i}\right)$ be labeled $v_{i, j}$. (See Fig. 7 for clarification.) For each $j \in\{1,2,3\}$, let $P_{i, j}$ be any directed path from a vertex in $\left[v_{i}\right]^{\phi^{-k} T}$ to a vertex in $\left[v_{i, j}\right]^{\phi^{-k} T}$, contained in $\left[e_{i, j}\right]^{\phi^{-k} T}$. Let the vertices along $P_{i, j}$ be labeled $\left\{w_{j_{1}}, \ldots, w_{j_{n}}\right\}$. If $w_{j_{1}}=w_{j_{k}}$ for $k>1$, delete all segments between $w_{j_{1}}$ and $w_{j_{k}}$. Continuing along the vertices in order, delete all cycles until $P_{i, j}$ has no selfintersections.

Since cells are convex, $\cap_{j}\left\{\left[e_{i, j}\right]^{\phi^{-k} T}\right\} \subset\left[v_{i}\right]^{\phi^{-k} T}$. Therefore any pair of $\left\{P_{i, 1}\right.$, $\left.P_{i, 2}, P_{i, 3}\right\}$ intersect only on $\left[v_{i}\right]^{\phi^{-k} T}$, if at all. Extend or delete edges of each $P_{i, j}$ until all three paths meet on a vertex of $\left[v_{i}\right]^{\phi^{-k} T}$, without overlapping on any edge of $\left[v_{i}\right]^{\phi^{-k} T}$. Assign $\Psi\left(v_{i}\right)=\cap_{j} P_{i, j}$, and let the outer endpoint of each path be labeled $v_{i, j}^{\prime}$.

We now map each partial edge $\overrightarrow{v_{i}, v_{i, j}}$ linearly to the path from $\Psi\left(v_{i}\right)$ to $v_{i, j}^{\prime}$. Let $k_{j}$ be the number of edges contained in the path from $\Psi\left(v_{i}\right)$ to $v_{i, j}^{\prime}$. Divide $\overrightarrow{v_{i}, v_{i, j}}$ into $k_{j}$ equal segments, and map each segment linearly to the corresponding edge on the path in $\phi^{-k} T$.

In this way $\Psi: \partial T \cap B_{\epsilon / 4}\left(v_{i}\right) \rightarrow \partial\left(\phi^{-k} T\right) \cap B_{\epsilon / 4}\left(v_{i}\right)$. Note that $|x-\Psi(x)|<\epsilon$ for all $x \in \partial T \cap B_{\epsilon / 4}\left(v_{i}\right)$, and that for any $l \in \operatorname{Im}\left(\partial T \cap B_{\epsilon / 4}\left(v_{i}\right)\right),\left.\Psi\right|_{\Psi^{-1}(l)}$ is linear and $\left|\Psi^{-1}(l)\right|<\epsilon / 4<d_{m} / 16$, and so this map meets the first two criteria of the proposition.

Extending $\Psi$ to all vertices in $\partial T$ 
Fig. 8 Constructing the image in $\phi^{-k} T$ of an edge in $T$

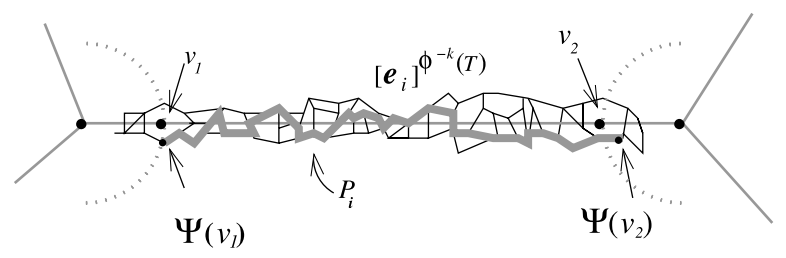

Next, we extend $\Psi$ to all $\partial T \cap B_{\epsilon / 4}(v)$, for all vertices $v \in T$. For each vertex $v$ in $T$, there is a unique $j \in\{1,2, \ldots, n\}$ and $g \in G$ such that $\left[B_{R_{k}+\epsilon / 4}(v)\right]^{T}=$ $g \cdot\left[B_{R_{k}+\epsilon / 4}\left(v_{j}\right)\right]^{T}$ and $v=g \cdot v_{j}$. Note that $g$ is unique since $T$ has no vertices which are loci of rotational symmetry of size $R_{k}$.

We define $\Psi$ on $\partial T \cap B_{\epsilon / 4}(v)$ as follows: if $w \in \partial T$ and $|w-v|<\epsilon / 4$, then let $\Psi(w)=g \Psi g^{-1}(w)$. Since $R_{k}$ is the radius of derivability of $\phi^{-k} T$ from $T$, we have that $\left[B_{\epsilon / 4}(v)\right]^{\phi^{-k} T}=g \cdot\left[B_{\epsilon / 4}\left(v_{j}\right)\right]^{\phi^{-k} T}$. Therefore, $g \Psi g^{-1}(w) \in \partial \phi^{-k} T$.

Defining $\Psi$ on edges in $T$

We will now partition the edges in $T$ according to their neighborhoods of radius $R_{k}+\epsilon / 2$. Let $\left\{e_{1}, \ldots, e_{m}\right\}$ be a maximal list of edges in $T$ such that $\left[N_{R_{k}+\epsilon / 2}\left(e_{i}\right)\right]^{T} \neq g \cdot\left[N_{R_{k}+\epsilon / 2}\left(e_{j}\right)\right]^{T}$ for any $i \neq j, g \in G$.

For a given edge $e_{i}$, we have already mapped under $\Psi$ the two segments of length $\epsilon / 4$ which are connected to each endpoint. Let $v_{1}$ and $v_{2}$ be the two points which are $\epsilon / 4$ away from each endpoint of $e_{i}$. (In other words, $\Psi\left(v_{1}\right)$ and $\Psi\left(v_{2}\right)$ were defined in the prior section.) (See Fig. 8 for clarification.)

We wish to map the line segment $\overrightarrow{v_{1}, v_{2}}$ to a path from $\Psi\left(v_{1}\right)$ to $\Psi\left(v_{2}\right)$, composed of edges in $\left[\overrightarrow{v_{1}, v_{2}}\right]^{\phi^{-k} T}$. Let $P_{i}$ be a path composed of edges in $\left[e_{i}\right]^{\phi^{-k} T}$ connecting $\Psi\left(v_{1}\right)$ and $\Psi\left(v_{2}\right)$. Let $v_{1}^{\prime}=\Psi\left(v_{1}\right), v_{n}^{\prime}=\Psi\left(v_{2}\right)$, and $\left\{v_{1}^{\prime}, v_{2}^{\prime}, \ldots, v_{n}^{\prime}\right\}$ be an ordered list of all the vertices on the path $P_{i}$, directed from $\Psi\left(v_{1}\right)$ to $\Psi\left(v_{2}\right)$. As before, we begin removing cycles by checking if $v_{1}^{\prime}=v_{j}^{\prime}$ for $j>1$, and if this occurs, deleting all segments between $v_{1}^{\prime}$ and $v_{j}^{\prime}$. We then repeat for the next available vertex until $P_{i}$ is not self-intersecting. Relabel the remaining vertices $\left\{v_{1}^{\prime}, \ldots, v_{n}^{\prime}\right\}$.

For each vertex $v_{j}^{\prime}$, choose a point $w_{j}$ on $e_{i}$, such that

1. $w_{1}=v_{1}, w_{n}=v_{2}$

2. $\left|w_{i}-v_{1}\right|<\left|w_{i+1}-v_{1}\right|$

3. $w_{j} \in\left\{\left[v_{j}^{\prime}\right]^{\phi^{-k} T} \cap e_{i}\right\}$ (which may contain disjoint segments of $e_{i}$ ).

Let $\Psi_{j}$ be the linear map from $\overrightarrow{w_{j}, w_{j+1}} \rightarrow \overrightarrow{v_{j}^{\prime}, v_{j+1}^{\prime}}$. Note that $\left|x-\Psi_{j}(x)\right| \leq$ $2 \lambda^{-k} d_{M}<\frac{\epsilon}{3}$. Concatenating the maps $\Psi_{j}$ creates a single, piecewise linear map $\Psi: \overrightarrow{w_{1}, w_{n}} \rightarrow \overrightarrow{v_{1}^{\prime}, v_{n}^{\prime}}$. Thus $\Psi: \overrightarrow{v_{1}, v_{2}} \rightarrow P_{i}$ satisfies the first two conditions of Proposition 34.

As before, we can now extend $\Psi$ to map all edges in $T$ to unions of edges in $\phi^{-k} T$, based on $\left[N_{R_{k}+\epsilon / 2}(e)\right]^{T}$. Define $\Psi(e)$ to be $g \cdot \Psi\left(e_{i}\right)$, where $e_{i} \in\left\{e_{1}, \ldots, e_{m}\right\}$ is the unique edge such that $\left[N_{R_{k}+\epsilon / 2}(e)\right]^{T}=g \cdot\left[N_{R_{k}+\epsilon / 2}\left(e_{i}\right)\right]^{T}$, and $g$ is unique since $e$ cannot contain any locus of rotational symmetry. Since the extension of $\Psi$ from $\left\{e_{1}, \ldots, e_{m}\right\}$ to all other edges is based on neighborhoods of radius $R_{k}+\epsilon / 2$, we have that $\Psi\left(e_{i}\right) \in \partial \phi^{-k} T$ implies $\Psi(e) \in \partial \phi^{-k} T$. 
Fig. 9 By choice of $k$, edges are separated by at least $3 \lambda^{-k} d_{M}$, outside of $B_{\epsilon / 4}(v)$

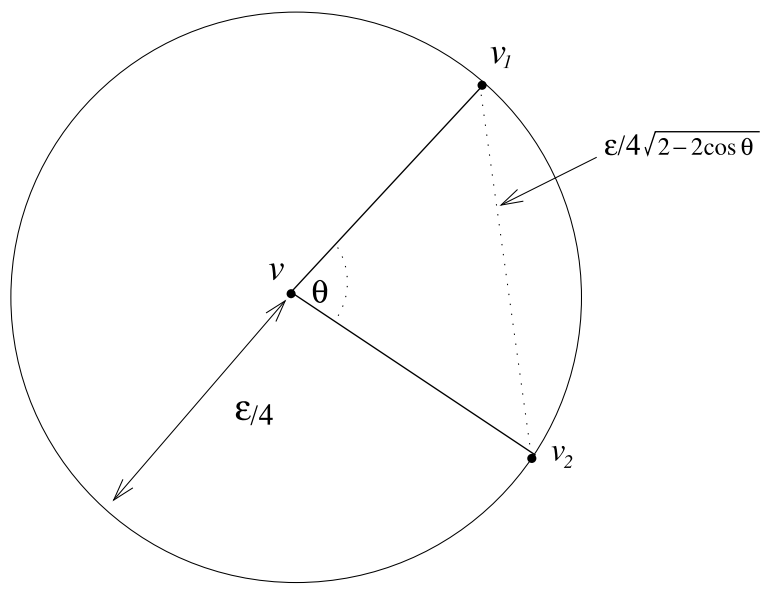

Next we show that the images of edges under $\Psi$ are non-intersecting. In $T$, suppose that two incoming edges to a vertex $v$ are separated by an angle $\theta, 0<\theta<\pi$. Let $v_{1}$ and $v_{2}$ be the two respective points on each edge which are $\epsilon / 4$ away from $v$. Then $\left|v_{1}-v_{2}\right|=\epsilon / 4 \sqrt{2-2 \cos \theta} \geq \epsilon / 4 \sqrt{2-2 \cos d_{\theta}}>3 \lambda^{-k} d_{M}$. This ensures that edges in $T$ which are outside of $\left[B_{\epsilon / 4}(v)\right]^{T}$ are separated by at least $3 \lambda^{-k} d_{M}$ (see Fig. 9) and so guarantees that the images of edges under $\Psi$ do not intersect. Thus $\Psi$ is injective.

By construction, $\Psi$ is continuous and linear on the preimage of all edges in $\operatorname{Im} \Psi$. We claim that $\Psi$ is locally derivable from $T$ with radius $\tilde{R}=R_{k}+\epsilon+d_{M}$. Suppose $\left[B_{R_{k}+\epsilon+d_{M}}(p)\right]^{T}=g \cdot\left[B_{R_{k}+\epsilon+d_{M}}(q)\right]^{T}$ for $p, q \in \partial T$. If $p$ and $q$ are within $\epsilon / 4$ of a vertices $v_{p}$ and $v_{q}$, respectively, then $\left[B_{R_{k}+3 \epsilon / 4}\left(v_{p}\right)\right]^{T}=g \cdot\left[B_{R_{k}+3 \epsilon / 4}\left(v_{q}\right)\right]^{T}$, which is more than sufficient to ensure that $\Psi(p)=g \cdot \Psi(q)$. Otherwise, we use the fact that $p$ and $q$ are contained within edges $e_{p}$ and $e_{q}$, and $\left[N_{R_{k}+\epsilon}\left(e_{p}\right)\right]^{T}=g \cdot\left[N_{R_{k}+\epsilon}\left(e_{q}\right)\right]^{T}$, which ensures that $\Psi(p)=g \cdot \Psi(q)$.

\section{Step 3: Constructing $\Psi_{\infty}$, a continuous, injective map from $\partial T$ to $\mathbb{R}^{2}$}

Define $\Psi_{n}:=\phi^{-n k}\left(\phi^{k} \Psi\right)^{n}$. In other words, $\Psi_{n}$ redraws edges in $T$ with edges in $\phi^{-n k} T$. Since $\phi$ and $\Psi$ are continuous and one-to-one, $\Psi_{n}$ is as well. Notice that since $\Psi: \partial T \rightarrow \partial\left(\phi^{-k} T\right)$, we have that $\phi^{k} \Psi: \partial T \rightarrow \partial T$, and so $\Psi_{n}: \partial T \rightarrow \partial \phi^{-n k} T$. With this, we define $\Psi_{\infty}: \partial T \rightarrow \mathbb{R}^{2}$ by $\Psi_{\infty}(x)=\lim _{n \rightarrow \infty} \Psi_{n}(x)$.

First we show that $\left\{\Psi_{n}\right\}$ converges uniformly:

$$
\begin{aligned}
\left|\Psi_{n}(x)-\Psi_{n+1}(x)\right| & =\left|\phi^{-n k}\left(\phi^{k} \Psi\right)^{n}(x)-\phi^{-(n+1) k}\left(\phi^{k} \Psi\right)^{(n+1)}(x)\right| \\
& =\left|\phi^{-n k}\left[\left(\phi^{k} \Psi\right)^{n}(x)-\phi^{-k}\left(\phi^{k} \Psi\right)^{(n+1)}(x)\right]\right| \\
& =\left|\phi^{-n k}\left[\left(\phi^{k} \Psi\right)^{n}(x)-\Psi\left(\phi^{k} \Psi\right)^{n}(x)\right]\right| \leq \lambda^{-n k} \epsilon .
\end{aligned}
$$

Therefore $\Psi_{\infty}(x)$ is well-defined.

$\Psi_{\infty}$ is injective 
We next claim that $\Psi_{\infty}$ is injective, for $\epsilon$ sufficiently small. Suppose $x, y \in \partial T$ and $\Psi_{\infty}(x)=\Psi_{\infty}(y)$.

Since $\left|\Psi_{n}(x)-\Psi_{n+1}(x)\right| \leq \lambda^{-n k} \epsilon,\left|x-\Psi_{\infty}(x)\right| \leq \frac{\epsilon}{1-\lambda^{-k}}<2 \epsilon$. (The inequality that $\lambda^{-k}<1 / 2$ follows from $\lambda^{-K}<\frac{d_{m}}{48 d_{M}} \sqrt{2-2 \cos d_{\theta}}$ and $k>K$.) Therefore $\mid x-$ $y \mid<4 \epsilon$. Since $\Psi_{n}=\phi^{-n k}\left(\phi^{k} \Psi\right)^{n}$, it follows that $\Psi_{n+1}=\phi^{-k} \Psi_{n} \phi^{k} \Psi$, and so

$$
\Psi_{\infty}=\phi^{-k} \Psi_{\infty} \phi^{k} \Psi
$$

Therefore we have $\phi^{-k} \Psi_{\infty} \phi^{k} \Psi(x)=\phi^{-k} \Psi_{\infty} \phi^{k} \Psi(y)$. Therefore, $\Psi_{\infty}\left[\phi^{k} \Psi(x)\right]=$ $\Psi_{\infty}\left[\phi^{k} \Psi(y)\right]$, and so resubstituting for $\Psi_{\infty} n$ times yields $\Psi_{\infty}\left[\left(\phi^{k} \Psi\right)^{n}(x)\right]=$ $\Psi_{\infty}\left[\left(\phi^{k} \Psi\right)^{n}(y)\right]$. Therefore $\left|\left(\phi^{k} \Psi\right)^{n}(x)-\left(\phi^{k} \Psi\right)^{n}(y)\right|<4 \epsilon$.

We can pick $\epsilon$ small enough that $x$ and $y$ must lie on the same or adjacent edges. Let $\epsilon$ be such that:

$$
4 \epsilon<\min \left\{\left|x_{1}-x_{2}\right|: x_{1}, x_{2} \text { lie on edges } e_{1}, e_{2} \text {, with } e_{1} \cap e_{2} \neq \emptyset .\right\} .
$$

This ensures that $\left(\phi^{k} \Psi\right)^{n}(x)$ and $\left(\phi^{k} \Psi\right)^{n}(y)$ lie on the same edge or adjacent edges in $\partial T$, since $\phi^{k} \Psi: \partial T \rightarrow \partial T$.

First, suppose that $\left(\phi^{k} \Psi\right)^{n}(x)$ and $\left(\phi^{k} \Psi\right)^{n}(y)$ lie on the same edge in $\partial T$. Since $\phi^{-k}$ takes edges to edges, and $\Psi$ takes edges to unions of edges, the preimage of any edge $e$ under $\Psi^{-1}$ must be contained within an edge of $T$. Therefore $\left(\phi^{k} \Psi\right)^{n-1}(x)$ and $\left(\phi^{k} \Psi\right)^{n-1}(y)$ lie on the same edge in $\partial T$. Repeating this, $\left(\phi^{k} \Psi\right)^{i}(x)$ and $\left(\phi^{k} \Psi\right)^{i}(y)$ will all lie on the same edge in $\partial T$ for all $i$, and so $x$ and $y$ lie on the same edge in $\partial T$.

$\Psi$ was constructed so that if $l$ is an edge in $\operatorname{Im} \Psi$, then the restriction $\left.\Psi\right|_{\Psi^{-1}(l)}$ is linear. Furthermore, there is a $\rho \in(0,1)$ such that if $e$ is an edge in $T$, and $l \in \Psi(e)$, then $\left|\Psi^{-1}(l)\right| \leq \rho|e|$.

Let $\hat{e}=\max \{|e|\}, e \in \partial T$. Then $\left|\Psi^{-1}(l)\right| \leq \rho \hat{e}$, for any edge $l$ in $\operatorname{Im} \Psi$, and so $|x-y| \leq \rho \hat{e}$. Therefore, the restriction $\left.\left(\phi^{k} \Psi\right)^{n}\right|_{\Psi^{-n}(l)}$ is linear, and $\left|\Psi^{-n}(l)\right| \leq \rho^{n}|e|$. Therefore, $|x-y| \leq \rho^{n} \hat{e}$, and so as $n \rightarrow \infty$, we have $x=y$.

Suppose that $\left(\phi^{k} \Psi\right)^{n}(x)$ and $\left(\phi^{k} \Psi\right)^{n}(y)$ lie on the adjacent edges in $\partial T$. The preimage under $\Psi$ of each pair of edges which share a vertex is each contained in edges which again share a vertex, and so we have that $\left(\phi^{k} \Psi\right)^{n-1}(x)$ and $\left(\phi^{k} \Psi\right)^{n-1}(y)$ lie on the adjacent edges in $\partial T$, and as above, eventually that $x$ and $y$ lie on adjacent edges in $\partial T$. As before, $\left|\Psi^{-1}(l)\right| \leq \rho \hat{e}$, for any edge $l$ in $\operatorname{Im} \Psi$, and so $|x-y| \leq$ $2 \rho \hat{e}$. Therefore $|x-y| \leq 2 \rho^{n} \hat{e}$, and so as $n \rightarrow \infty$, we have $x=y$. Therefore $\Psi_{\infty}$ is injective.

\section{Step 4. Defining a tiling from $\operatorname{Im} \Psi_{\infty}$, called $T_{\infty}$, which is MLD to $T$}

Let $t_{i}=\left(A_{i}, l_{i}\right)$ be a tile in $T$. Since $\Psi_{\infty}$ is injective, by the Jordan Curve theorem, $\Psi_{\infty}$ maps $\partial A_{i}$ to a simple curve which separates the plane into two components, of which $\Psi_{\infty}\left(\partial A_{i}\right)$ is the boundary. We will now define a tiling, $T_{\infty}$, such that the closure of the support of the interior of $\Psi_{\infty}\left(\partial A_{i}\right)$ is the support for a tile in $T_{\infty}$. Let this be called $A_{i}^{\prime}$, the compact set for a tile $t_{i}^{\prime}$ in $T_{\infty}$.

We label $\left\{t_{i}^{\prime}\right\}$ based on a neighborhood of radius $\tilde{R}=R_{\Psi}$, the radius of derivability of $\Psi$. Formally, let $\left\{\left[N_{\tilde{R}}\left(t_{i}\right)\right]^{T}\right\}$ be a comprehensive, finite list of neighborhoods of 
tiles in $T$ of radius $\tilde{R}$, up to action by $G$, where $t_{i}$ has support $A_{i}$. Let $l_{i}^{\prime}=\left[N_{\tilde{R}}\left(t_{i}\right)\right]^{T}$. Given an unlabeled tile $t^{\prime}$ in $T_{\infty}$, let $t$ be the associated tile in $T$ such that $\Psi_{\infty}: \partial t \rightarrow$ $\partial t^{\prime}$. We label $t^{\prime}$ with $l_{i}^{\prime}$ if $\left[N_{\tilde{R}}(t)\right]^{T}=g \cdot\left[N_{\tilde{R}}\left(t_{i}\right)\right]^{T}$ for some $g \in G$.

Next we must show that the image of tiles of $T$ under $\Psi_{\infty}$ overlap only on their boundaries. Let $A_{i}$ be the support of a tile in $T$, and $\Psi_{\infty}\left(\partial A_{i}\right)$ be the corresponding compact set in $T_{\infty}$. Since $\left|x-\Psi_{\infty}(x)\right|<4 \epsilon$ for $x \in \partial T$, the unbounded component of $\partial T \backslash \partial A_{i}$ must overlap with the unbounded component of $\mathbb{R}^{2} \backslash \Psi_{\infty}\left(\partial A_{i}\right)$.

Since $\partial T \backslash \partial A_{i}$ is connected, so is $\Psi_{\infty}\left(\partial T \backslash \partial A_{i}\right)$. And since $\Psi_{\infty}$ is injective, $\Psi_{\infty}\left(\partial T \backslash \partial A_{i}\right)$ must lie entirely in the unbounded component of $\mathbb{R}^{2} \backslash \Psi_{\infty}\left(\partial A_{i}\right)$. If $A_{j}$ is the support of a different tile in $T$, then $\operatorname{int} A_{j} \subset \mathbb{R}^{2} \backslash A_{i}$, and so $\operatorname{int}\left(\Psi_{\infty}\left(\partial A_{j}\right)\right)$ is contained in the unbounded component of $\mathbb{R}^{2} \backslash \Psi_{\infty}\left(\partial A_{i}\right)$. Therefore, $\operatorname{int}\left(\Psi_{\infty}\left(\partial A_{j}\right)\right) \cap$ $\operatorname{int}\left(\Psi_{\infty}\left(\partial A_{i}\right)\right)=\emptyset$.

Lastly we must show that the tiles cover $\mathbb{R}^{2}$. Let $z \in \mathbb{R}^{2}$. Then $\left[B_{d_{M}+4 \epsilon}(z)\right]^{T}$ is the union of finitely many tiles of $T$, and $z$ lies in the bounded component of $\Psi_{\infty}\left(\partial\left[B_{d_{M}+4 \epsilon}(z)\right]^{T}\right)$. Since $\partial\left[B_{d_{M}+4 \epsilon}(z)\right]^{T}$ is a compact set, $\left.\Psi_{\infty}\right|_{\partial\left[B_{d_{M}+4 \epsilon}(z)\right]^{T}}$ can be extended to a homeomorphism $h:\left[B_{d_{M}+4 \epsilon}(z)\right]^{T} \rightarrow \operatorname{supp}\left(\operatorname{int} \Psi_{\infty}\left(\partial\left[B_{d_{M}+4 \epsilon}(z)\right]^{T}\right)\right)$. Therefore $z$ is in the image under $h$ of some tile $t \in\left[B_{d_{M}+4 \epsilon}(z)\right]^{T}$, and so $T_{\infty}$ does in fact cover $\mathbb{R}^{2}$.

We have shown that $T_{\infty}$ is a cover of $\mathbb{R}^{2}$ by tiles which overlap only on their boundaries, and so $T_{\infty}$ is a tiling.

\section{Theorem $35 T_{\infty}$ and $T$ are MLD tilings, for $\epsilon$ sufficiently small.}

$T_{\infty}$ is locally derivable from $T$

Let $\rho$ be the recognizability radius of $T$ from $\phi T$. Note that $\Psi$ is locally derivable under any radius larger than $\tilde{R}$. Without loss of generality, we may assume that $\tilde{R}>$ $\frac{\lambda^{k}(\rho+\epsilon)}{\lambda^{k}-1}$.

Lemma $36 \Psi_{n}$ is locally derivable from $T$ with radius $\tilde{R}$, for all $n$.

We will proceed by induction; $\Psi=\Psi_{1}$ is locally derivable with radius $\tilde{R}$ by construction. Assume $\Psi_{n}$ is as well.

Suppose $\left[B_{\tilde{R}}(x)\right]^{T}=g \cdot\left[B_{\tilde{R}}(y)\right]^{T}$, with $x=g \cdot y$. Since $|\Psi(x)-x|<\epsilon$, $\left[B_{\tilde{R}-\epsilon}(\Psi(x))\right]^{T}=g \cdot\left[B_{\tilde{R}-\epsilon}\left(g^{-1} \cdot \Psi(x)\right)\right]^{T}$. Applying $\phi^{k}$, we have $\left[B_{\lambda^{k}(\tilde{R}-\epsilon)} \times\right.$ $\left.\left(\phi^{k} \Psi(x)\right)\right]^{\phi^{k} T}=\phi^{k} g \phi^{-k} \cdot\left[B_{\lambda^{k}(\tilde{R}-\epsilon)}\left(\phi^{k}\left(g^{-1} \cdot \Psi(x)\right)\right)\right]^{\phi^{k} T}$.

Since $\lambda^{k} \rho$ is the radius of derivability of $T$ from $\phi^{k} T,\left[B_{\lambda^{k}(\tilde{R}-\epsilon)-\lambda^{k} \rho}\left(\phi^{k} \Psi(x)\right)\right]^{T}=$ $\phi^{k} g \phi^{-k} \cdot\left[B_{\lambda^{k}(\tilde{R}-\epsilon)-\lambda^{k} \rho}\left(\phi^{k}\left(g^{-1} \cdot \Psi(x)\right)\right)\right]^{T}$. Now, since $\tilde{R}$ is the radius of derivability of $\Psi$ from $T$, and $\left[B_{\tilde{R}}(x)\right]^{T}=g \cdot\left[B_{\tilde{R}}(y)\right]^{T}$, we have that $\Psi(x)=g \cdot \Psi(y)$. Therefore, $\left[B_{\lambda^{k}(\tilde{R}-\epsilon)-\lambda^{k} \rho}\left(\phi^{k} \Psi(x)\right)\right]^{T}=\phi^{k} g \phi^{-k} \cdot\left[B_{\lambda^{k}(\tilde{R}-\epsilon)-\lambda^{k} \rho}\left(\phi^{k}(\Psi(y))\right)\right]^{T}$.

By the inductive hypothesis, $\Psi_{n}$ is derivable from $T$ with radius $\tilde{R}$. Since $\lambda^{k}(\tilde{R}-$ $\epsilon)-\lambda^{k} \rho>\tilde{R}$, we have that $\left.\Psi_{n} \phi^{k} \Psi(x)\right)=\phi^{k} g^{-1} \phi^{-k} \cdot \Psi_{n}\left(\phi^{k}(\Psi(y))\right)$. Since $\Psi_{n+1}=$ $\phi^{-k} \Psi_{n} \phi^{k} \Psi$, we get that $\phi^{k} \Psi_{n+1}(x)=\phi^{k} g^{-1} \Psi_{n+1}(y)$, and so $\Psi_{n+1}$ is also locally derivable with radius $\tilde{R}$. 
Once we have that all $\Psi_{n}$ are LD from $T$ with radius $\tilde{R}$, it follows that $\lim _{n \rightarrow \infty} \Psi_{n}=\Psi_{\infty}$ is as well.

Now we must show that $T_{\infty}$ is LD from $T$. Suppose $\left[B_{\tilde{R}+d_{M}+2 \epsilon}(x)\right]^{T}=g$. $\left[B_{\tilde{R}+d_{M}+2 \epsilon}(y)\right]^{T}$, with $x=g \cdot y$. For every $p \in \partial[x]^{T}$ and $q \in \partial[y]^{T}$ with $q=g \cdot p$, we have $\Psi_{\infty}(p)=g \cdot \Psi_{\infty}(q)$. Therefore $\Psi_{\infty}\left(\partial[x]^{T}\right)=g \cdot \Psi_{\infty}\left(\partial[y]^{T}\right)$. Since tiles in $T_{\infty}$ were labeled based on a neighborhood of $\tilde{R}$ in $T,[x]^{T_{\infty}}$ and $[y]^{T_{\infty}}$ have the same label. Therefore $[x]^{T_{\infty}}=g \cdot[y]^{T_{\infty}}$.

$T$ is locally derivable from $T_{\infty}$

First, we must ensure that tiles in $T_{\infty}$ do not have any additional rotational symmetry that tiles in $T$ lack. If $t$ is a tile in $T$, let $\Psi_{\infty}(t)$ refer to the tile in $T_{\infty}$ whose boundary is the image of the boundary of $t$ under $\Psi_{\infty}$.

Suppose $t \neq g \cdot t$ but $\Psi_{\infty}(t)=\Psi_{\infty}(g \cdot t)$. Let $\left\{v_{1}, \ldots, v_{n}\right\}$ be the ordered vertices of $t$, and $\left\{\Psi_{\infty}\left(v_{1}\right), \ldots, \Psi_{\infty}\left(v_{n}\right)\right\}$ be the corresponding images under $\Psi_{\infty}$. Then $\left\{\Psi_{\infty}\left(v_{1}\right), \ldots, \Psi_{\infty}\left(v_{n}\right)\right\}=\left\{\Psi_{\infty}\left(g \cdot v_{1}\right), \ldots, \Psi_{\infty}\left(g \cdot v_{n}\right)\right\}$

Let $v_{i}$ be a vertex of $t$ such that $g \cdot v_{i} \neq v_{j}$ for any $j$, but $\Psi_{\infty}\left(g \cdot v_{i}\right)=\Psi_{\infty}\left(v_{j}\right)$ for some $j$. Let $d:=\left|g \cdot v_{i}-v_{j}\right|$. Let $\hat{d}$ be the minimum $d$ for any vertex in any tile $t$ which lacks symmetry that $\Psi_{\infty}(t)$ has. If we take $\epsilon<\hat{d} / 3$, then we ensure that $\Psi_{\infty}\left(g \cdot v_{i}\right) \neq \Psi_{\infty}\left(v_{j}\right)$, and so $T_{\infty}$ will only have rotational symmetry if $T$ does.

Suppose $\left[B_{2 d_{M}+\epsilon}(x)\right]^{T_{\infty}}=g \cdot\left[B_{2 d_{M}+\epsilon}(y)\right]^{T_{\infty}}$ and $x=g \cdot y$. While it may be that $\Psi\left([x]^{T}\right) \neq[x]^{T_{\infty}}$ and $\Psi\left([y]^{T}\right) \neq[y]^{T_{\infty}}$, we still have that $\Psi\left([x]^{T}\right) \in\left[B_{2 d_{M}+\epsilon}(x)\right]^{T_{\infty}}$ and $\Psi\left([y]^{T}\right) \in\left[B_{2 d_{M}+\epsilon}(y)\right]^{T_{\infty}}$, and so $\Psi\left([x]^{T}\right)$ and $\Psi\left([y]^{T}\right)$ have matching labels in $T_{\infty}$. Therefore $\left[N_{\tilde{R}}\left([x]^{T}\right)\right]^{T}=g^{\prime} \cdot\left[N_{\tilde{R}}\left([y]^{T}\right)\right]^{T}$ for some $g^{\prime} \in G$, and so $[x]^{T}=$ $g^{\prime} \cdot[y]^{T}$. Since $x=g \cdot y, g^{\prime} \neq g$ only if $[x]^{T_{\infty}}$ and $[y]^{T_{\infty}}$ have a rotational symmetry which $[x]^{T}$ and $[y]^{T}$ lack. By choice of $\epsilon$ this cannot occur, and so $g^{\prime}=g$.

Therefore $T$ and $T_{\infty}$ are MLD tilings.

\section{Step 5. $T_{\infty}$ is a self-similar tiling; $\bar{T}_{\infty}$ is a self-similar tiling space which is MLD to $X$}

\section{Theorem $37 T_{\infty}$ is a self-similar tiling.}

We must show first that for a tile $t \in T_{\infty}, \phi^{k}(t)$ is the union of tiles in $\phi^{k} T_{\infty}$.

From (1), in Step 3, $\Psi_{\infty}=\phi^{-k} \Psi_{\infty} \phi^{k} \Psi$. Therefore $\phi^{k}\left(\Psi_{\infty}(\partial T)\right) \subset \Psi_{\infty}(\partial T)$. If we take the support $A$ of a tile $t$ in $T_{\infty}$, we have that $\partial A$ is a Jordan curve, and so $\phi^{k}(\partial A)$ divides $\mathbb{R}^{2}$ into two components. Since $\phi^{k} \partial A \subset \partial T_{\infty}$, the support $A^{\prime}$ of any tile $t^{\prime}$ in $T_{\infty}$ must lie entirely in one of the two components of $\mathbb{R}^{2} \backslash \phi^{k} \partial A$. Therefore $\phi^{k} \partial A$ is exactly the union of all tiles whose interiors have nontrivial intersection with the interior of $\phi^{k} \partial A$.

Second, we must show that if $t_{1}$ and $t_{2}$ are tiles in $T_{\infty}$ with $t_{1}=g \cdot t_{2}$, then $\phi^{k}\left(t_{1}\right)=$ $g^{\prime} \cdot \phi^{k}\left(t_{2}\right)$ as patches of tiles in $\phi^{k} T$, where $g^{\prime}=\phi^{k} g \phi^{-k}$.

Suppose that $t_{1}, t_{2}$ are tiles in $T_{\infty}$ with the same label, and $t_{1}=g \cdot t_{2}$. Since the labels are based on a radius $\tilde{R}$ of the corresponding tile in $T$, we have that 
$\left[N_{\tilde{R}}\left(\Psi_{\infty}^{-1}\left(t_{1}\right)\right)\right]^{T}=g \cdot\left[N_{\tilde{R}}\left(\Psi_{\infty}^{-1}\left(t_{2}\right)\right)\right]^{T}$. Applying $\phi^{k}$, we have that

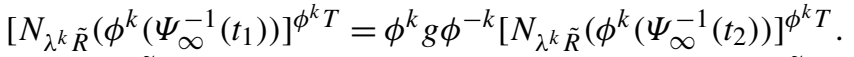

Since $\tilde{R}$ is the radius of derivability of $\Psi_{\infty}$ onto $T, \lambda^{k} \tilde{R}$ is a lower bound on the local derivability radius of $\phi^{k} \Psi_{\infty}$ onto $\phi^{k} T$. Therefore, for all points $[x] \in \phi^{k}\left(\Psi_{\infty}^{-1}\left(t_{1}\right)\right)$ and $[y] \in \phi^{k}\left(\Psi_{\infty}^{-1}\left(t_{2}\right)\right)$ with $x=\phi^{k} g \phi^{-k} y,[x]^{\phi^{k}} T_{\infty}=\phi^{k} \cdot g \cdot \phi^{-k}[y]^{\phi^{k} T_{\infty}}$.

Therefore $T_{\infty}$ is a self-similar tiling.

Theorem 26 If $X$ is a pseudo-self-similar tiling space, then it is MLD to a self-similar tiling space, $X^{\prime}$.

Proof Let $T$ be a pseudo-self-similar tiling contained in $X$. Then $T_{\infty}$ is self-similar, and $\bar{T}_{\infty}$ is MLD to $X$.

\section{Conclusion}

We have shown, up to MLD, a series of results detailing the relationship between self-similar tiling spaces and pseudo-self-similar spaces, and derived Voronoi spaces, in $\mathbb{R}^{2}$. First, a tiling is MLD to all of its derived Voronoi tilings, and likewise for tiling spaces. Next, given a self-similar tiling $T$, the associated set $\left\{V_{(T, \overrightarrow{0}, r)}\right\}$ is $\sigma$ finite, which implies that a self-similar tiling must contain a scaled pair of tilings, and that a self-similar tiling space must contain a scaled pair of orbits. Having a scaled pair of tilings or orbits, in turn, implies that the underlying tiling or tiling space is pseudo-self-similar. Finally, we've shown that a pseudo-self-similar tiling or tiling space is MLD to a self-similar tiling or tiling space.

These results couch self-similarity and pseudo-self-similarity in a context where the group structure is utilized. While many tilings whose group includes only finite order rotations group can be redefined as tilings whose group is strictly translations, much of the basic structure of the tiling is lost in this relabeling. Second, tilings whose group includes infinite order rotations cannot be dealt with at all, without incorporating rotations into the discussion. Finally, including tiling spaces in these results allows for a broader range of connections with features of tiling spaces such as their dynamics or their topology, which does not exist with individual tilings.

There are large impediments to extending these results to higher dimensions. We often use the fact that self-similar tilings are recognizable, which is not necessarily true in dimension 3 and higher. Holton et al. [1] have an example of a pseudoself-similar tiling of $\mathbb{R}^{3}$ which is not recognizable. It would be interesting to explore whether there are self-similar tilings of $\mathbb{R}^{3}$ which are not recognizable, or whether there are pseudo-self-similar tilings of $\mathbb{R}^{3}$ which are not MLD to self-similar tilings.

Acknowledgements I would like to acknowledge first my advisor, Lorenzo Sadun, for many hours and much guidance given in writing this paper. Also, the referee provided much indispensable help revising and improving this paper. This was made possible by funding from NSF Grant 26-1136-4712, via Karen Uhlenbeck and the geometry group at the University of Texas. 


\section{References}

1. Holton, C., Radin, C., Sadun, L.: Conjugacies for tiling dynamical systems. Commun. Math. Phys. 254, 343-359 (2005)

2. Priebe, N.: Towards a characterization of self-similar tilings in terms of derived Voronoi tessellations. Geom. Dedic. 79, 239-265 (2000)

3. Priebe, N., Solomyak, B.: Characterization of planar pseudo-self-similar tilings. Discrete Comput. Geom. 26, 289-306 (2001) 